\title{
Rejets \\ des produits de dragages à l'aval d'un barrage sur l'Oued Hamiz
}

PAR

I. Valembois

Conseiller scientifique

Direction des Etudes et Recherches

d'Electricité de France
ET

\author{
C. Migniot \\ Ingénieur en chef \\ au Département de Sédimentologie \\ Laboratoire Centeal d'Hydraulique de France \\ Maisons-Alfort
}

L'évacuation jusqu'à la mer, par le seul jeu des courants, des matériaux provenant d'une retenue de barrage, doit être examinée avec soin avant d'entreprendre une campagne de dévasement.

Il ne suffit pas en effet de mettre au point uniquement une technologie de dragage par des méthodes classiques, ou par des hydro-aspirateurs branchés sur des orifices de chasse, mais de définir le débit liquide minimum à utiliser pour transporter le maximum de sédiments dans les différents tronçons de la rivière en aval du barrage. En s'écartant trop fortement de l'équilibre susceptible d'exister entre les paramètres hydrauliques et sédimentologiques, on risque d'entrainer un colmatage important de la rivière ou, au contraire, une perte d'eau préjudiciable à son utilisation.

Une étude de la capacité de transport de la rivière, en aval du barrage, permet d'aborder ce problème en se basant d'une part sur la nature des matériaux accumulés dans la retenue et sur leurs propriétés physiques, d'autre part sur des considérations théoriques et des recherches sur la morphologie des fonds de la rivière après différents débits ou chasses du barrage.

Compte tenu des débits liquides disponibles, des ordres de grandeur des débits solides peuvent alors être avancés pour les différents types de matériaux (vase, sable, graviers, galets de vase...) et dans les différents tronçons de la rivière permettant de planifier le rythme des dragages à entreprendre pour redonner au barrage une capacité suffisante, tout en maintenant un volume d'eau nécessaire pour les utilisations agraires ou industrielles pendant la saison sèche.

Les recherches entreprises par le Laboratoire Central d'Hydraulique de France (LCHF) en 1966 sur le barrage de Hamiz en Algérie (1) illustrent les résultats que l'on peut

(1) Etudes effectuées pour le comple du Service des Etudes Générales et Grands Travaux Hydrauliques d'Algérie (SEGGTH). qui a participé d'une façon très active aux différentes mesures effectuées en nature (1955 1966). obtenir dans ce domaine et la façon dont ce problème a été abordé en utilisant diverses méthodes, dont certaines classiques (2) et d'autres ont été développées à l'occasion de ces études, le premier auteur assurant alors la direction technique du ILCHF.

Toutes ces méthodes permettent de conclure avec assez de sécurité à la possibilité ou à l'impossibilité d'évacuer telle ou telle fraction du dépôt sédimentaire. Il ne faudrait cependant pas s'illusionner sur leur précision et considérer comme ayant une valeur absolue des chiffres qui ne sont que des ordres de grandeurs.

\section{1 - Situation et̂́ caractéristiques du barrage du Hamiz. Son régime hydrologique et les besoins en eau}

\section{1 - SITUATION ET CARACTÉRISTIQUES}

Le barrage du Hamiz, utilisé principalement pour l'irrigation de la Mitidja, est situé à $35 \mathrm{~km}$ au sud-est d'Alger (fig. 1). Réalisé entre 1869 et 1884 , ce barrage a été surélevé en 1935 pour atteindre une hauteur de $45 \mathrm{~m}$ et une longueur au sommet de $170 \mathrm{~m}$ environ. Le réservoir avait une capacité initiale de 30 millions de $\mathrm{m}^{3}$ qui a été ramenée progressivement à moins de 13 millions de $\mathrm{m}^{\hat{3}}$ en

(2) «Etude bibliographique et théorique de la capacité de transport des sédiments par les fleuves et les rivières». Etude faite par le LCHF pour le compte du Secrétariat d'Etat aux Afraires Etrangères. Direction de l'Aide au Déve'oppenent (1966). 


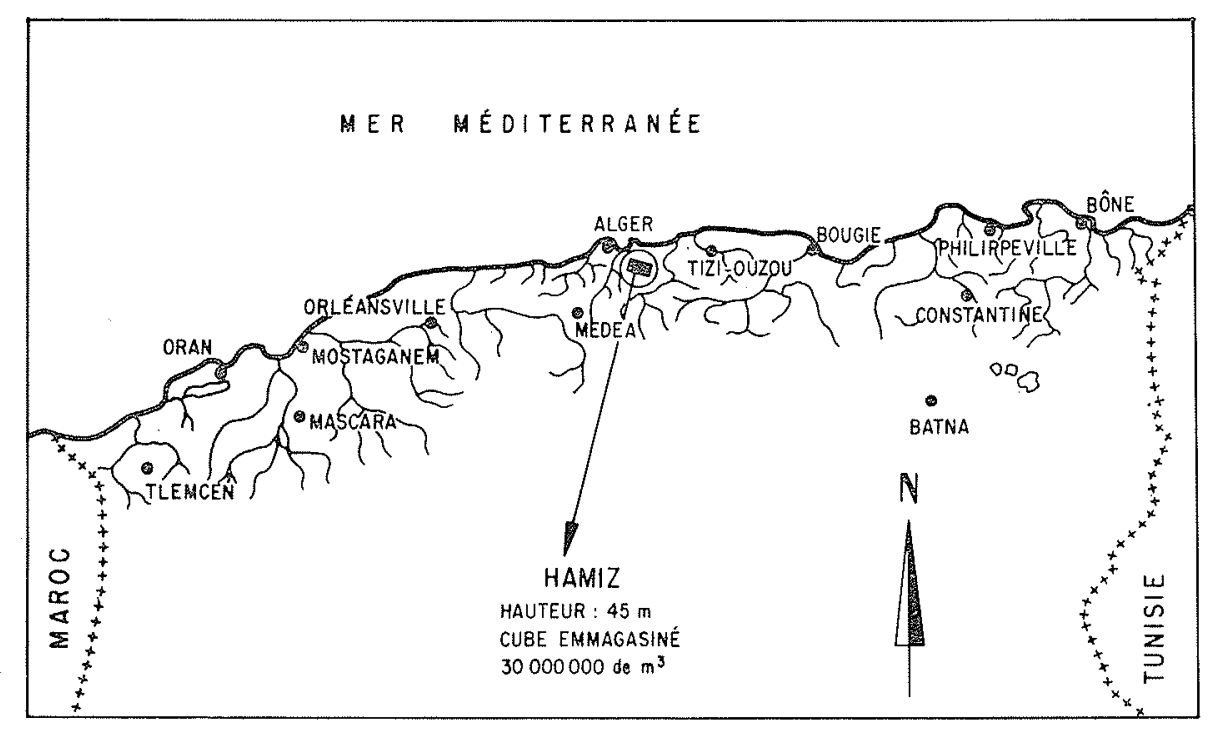

$1 /$

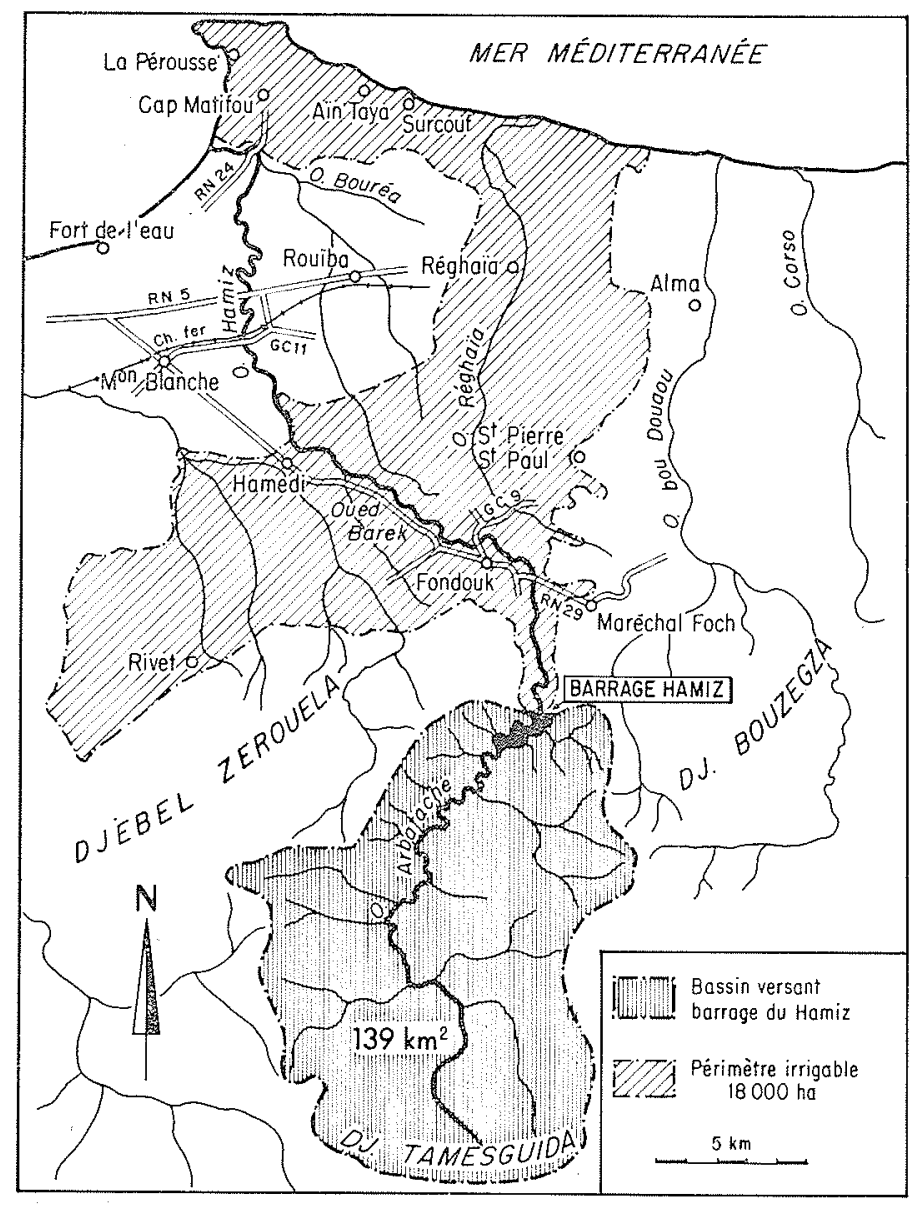

2/ Bassin versant du Hamiz et périmètre irrigable.

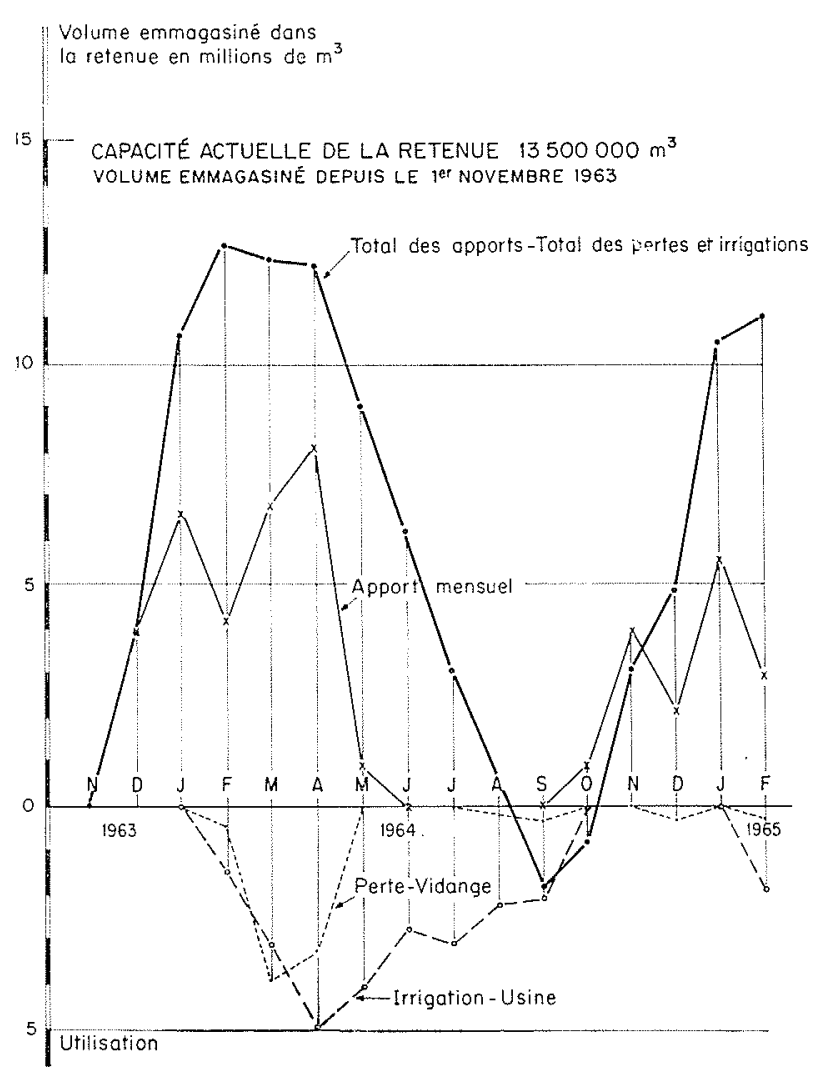

3/ Apports et utilisation de l'eau du barrage en 1964 (année de faible pluviosité). 


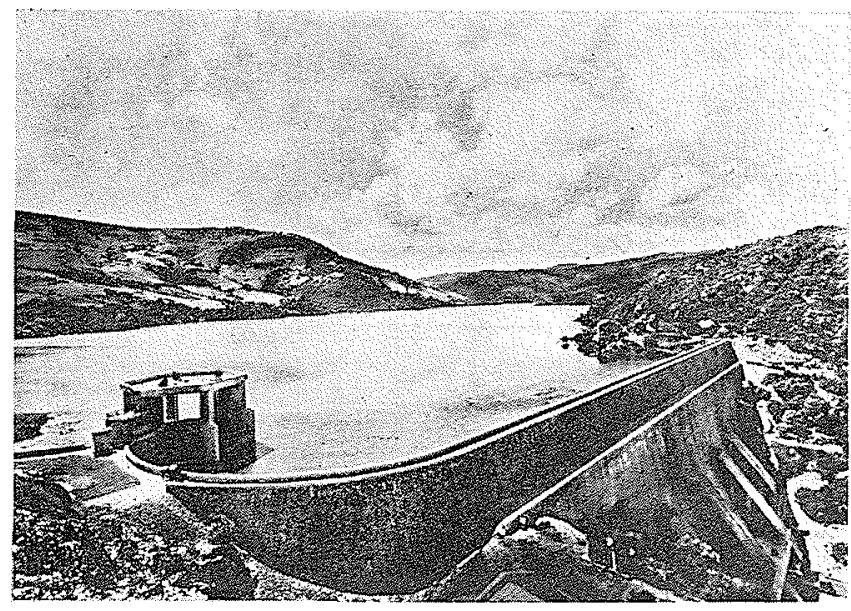

4/ Le plan d'eau après la construction du barrage.

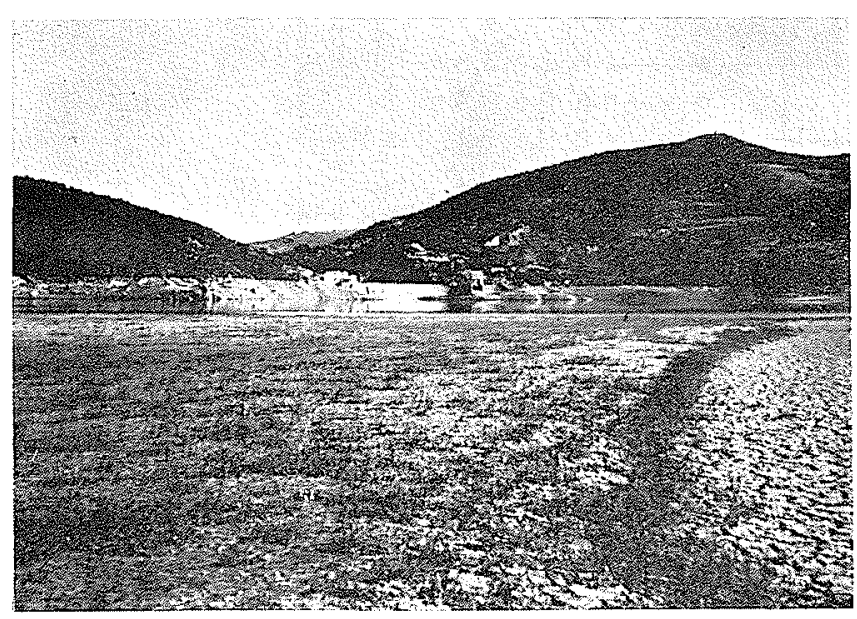

5/ La retenue en 1964.

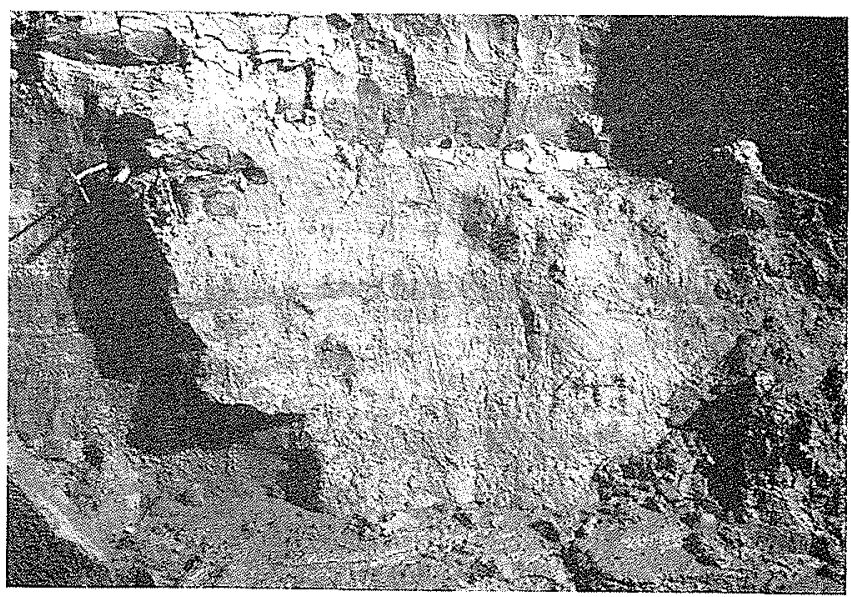

6/ Feuillets sédimentaires à l'amont de la retenue.
1964 par suite d'une sédimentation annuelle de 200000 à $250000 \mathrm{~m}^{3}$ (1/10" environ de celle du barrage de l'Oued Fodda).

La superficie du bassin versant de l'Oued Hamiz, en amont du barrage, est de $140 \mathrm{~km}^{2}$ avec une longueur de $16 \mathrm{~km}$ environ et une altitude maximale de $1140 \mathrm{~m}$ (fig. 2). Ce bassin est constitué en quasi totalité par des sédiments marneux et comprend trois terrains principaux: le flysch alboaptien, le sénonien formé de marnes avec lentilles calcaires et le flysch de l'éocène supérieure.

Ces terrains peuvent, s'ils ne sont pas suffisamment protégés, se dégrader sous l'action des pluies et libérer un stock important de sédiments dont une partie seulement se dépose dans la retenue, le reste étant évacué au cours des crues par le déversoir, dont la crête située à $5 \mathrm{~m}$ en dessous du barrage permet d'assurer un débit de $750 \mathrm{~m}^{3} / \mathrm{s}$.

\section{2 - RÉGIME HYDROLOGIQUE ET BESOINS EN EAU}

La connaissance des données hydrologiques est un des premiers éléments indispensables pour définir les quantités d'eau susceptibles d'être utilisées au moment des dragages pour augmenter la capacité de transport de l'oued en aval du barrage.

L'étude de la pluviosité montre que les précipitations atteignent, en moyenne, $850 \mathrm{~mm}$ par an sur le bassin versant du Hamiz, les écarts pouvant être compris entre $550 \mathrm{~mm}$ et $1400 \mathrm{~mm}$. Les pluies sont particulièrement abondantes de novembre à février où elles dépassent $100 \mathrm{~mm}$ par mois. La saison sèche s'étend de juin à septembre.

Le coefficient de ruissellement, qui n'est autre que le pourcentage d'apport d'eau dans la retenue rapporté au volume de pluie tombé sur la totalité du bassin, est en moyenne de $43,5 \%$; il crôt avec la pluviosité et passe de $32 \%$ pour une pluviosité annueile de $550 \mathrm{~mm}$ à $69 \%$ pour une pluviosité de $1300 \mathrm{~mm}$. L'apport de ruissellement du Hamiz est donc assez élevé par rapport aux autres bassins algériens.

Les apports d'eau dans la retenue sont, en moyenne, de 52 millions de $\mathrm{m}^{3}$ par an (moyenne des trente dernières années) et peuvent varier d'une année à l'autre entre 20 millions de $m^{3}$ et 100 millions de $\mathrm{m}^{3}$ par an. Le débit moyen hebdomadaire peut atteindre $50 \mathrm{~m}^{3} / \mathrm{s}$ avec des valeurs de pointe instantanée beaucoup plus considérables.

Compte tenu de la capacité actuelle de la retenue (13 millions de $\mathrm{m}^{3}$ en 1964) le barrage du Hamiz ne retient donc que $25 \%$ des apports liquides annuels alors qu'il en retenait $60 \%$ immédiatement après sa construction.

Les besoins en eau, utilisée principalement pour l'irrigation, atteignent 15 à 20 millions de $\mathrm{m}^{3}$ par an, soit $30 \%$ des apports liquides.

A ce chiffre il faut ajouter $3 \%$ de pertes d'eau.

En faisant le bilan des apports d'eau, des besoins pour l'irrigation et des pertes, on constate que le barrage est plein entre les mois de février et d'avril et se trouve à sec entre août et octobre (fig. 3). Dès 1964 la situation était donc devenue critique et interdisait tout développement agraire dans la plaine de la Mitidja si l'on n'entreprenait pas très rapidement des travaux de dévasement. 


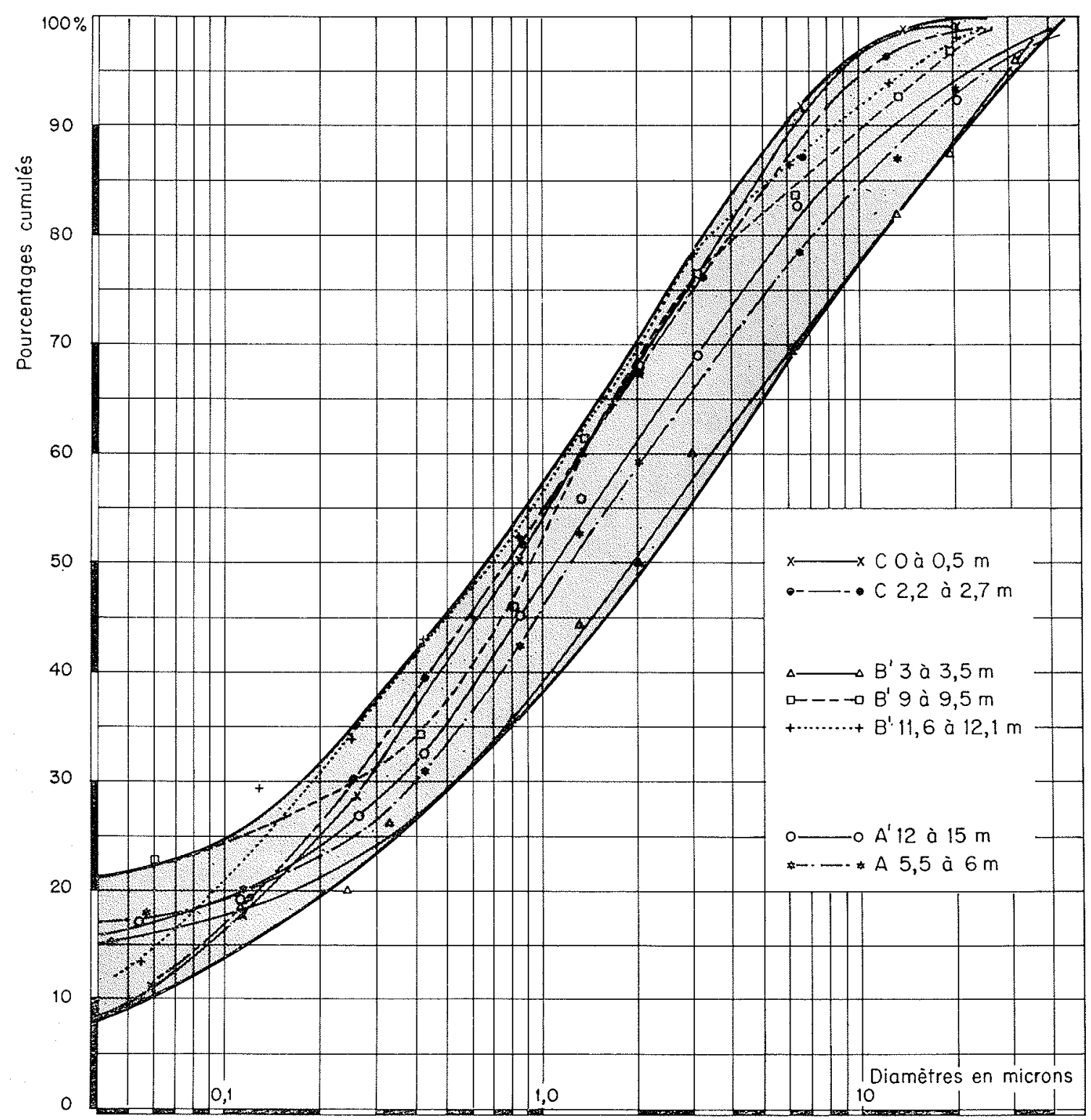

7/

Courbes

granulométriques

des vases.

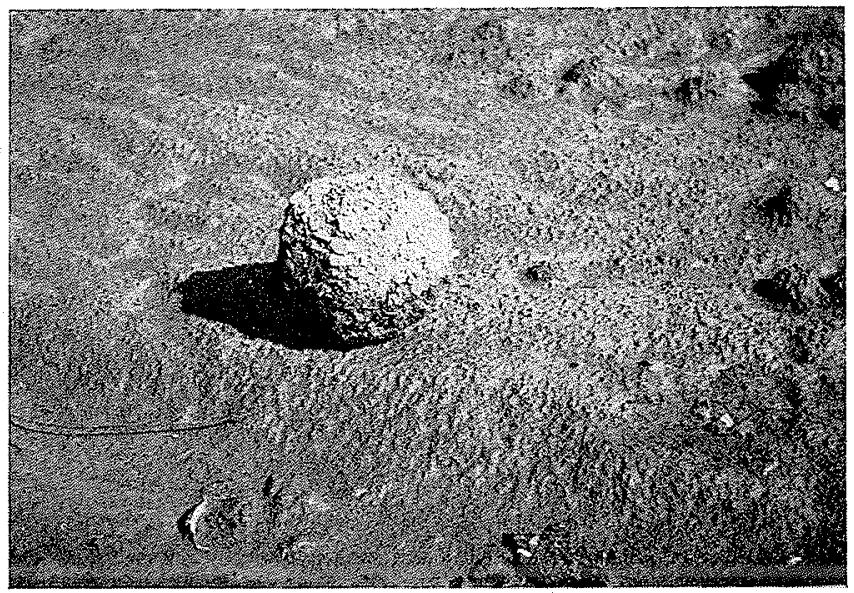

8/ Un galet de vase. 


\section{2 - Importance et nature des sédiments accumulés dans la retenue. Leurs pro- priétés physiques et leurs comportements sous les actions hydrodynamiques}

\section{1 - IMPORTANCE ET NATURE DES SÉDIMENTS}

Offrant un plan d'eau de près de 1500 ha quelques années après sa construction (fig. 4), la retenue du barrage du Hamiz présentait en 1964 l'aspect d'une immense vasière (fig. 5) avec une épaisseur de sédiments variant entre 20 et $25 \mathrm{~m}$ dans l'axe de la retenue.

Les forages effectués montrent que les sédiments déposés dans la retenue sont constitués presque exclusivement de vases argileuses sur les 700 premiers mètres en amont du barrage, les éléments sableux n'apparaissant qu'à proximité de l'ancien lit de l'oued et dans la partie très amont de la retenue où la granulométrie varie considérablement avec la profondeur et où l'on constate de véritables «feuillets » sédimentaires qui caractérisent vraisemblablement un régime particulier de l'oued (fig. 6).

Les vases $\left(^{3}\right)$ sont extrêmement fines et le diamètre moyen de la fraction détritique est de 1 micron; en tenant compte de la fraction calcaire qui représente $20 \%$, le diamètre moyen serait de 2 microns. Ces vases contiennent très peu d'humates $(0,1 \%$ à l'aval et $0,6 \%$ en amont) et leur teneur en eau, en place, varie entre 45 et $55 \%$, ce qui correspondrait à des densités comprises entre 1,6 et 1,7 (fig. 7).

Les sables sont constitués par deux groupes, le premier ayant des diamètres moyens de 0,06 à $0,1 \mathrm{~mm}$, et le second des diamètres moyens de 0,4 à $0,5 \mathrm{~mm}$ avec un maximum de $1,5 \mathrm{~mm}$.

A ces sables peuvent s'incorporer des graviers et des galets, d'ailleurs en petit nombre, ainsi que des galets de vase qui se forment au cours du charriage d'éléments de sols argileux accumulés aux rives (fig. 8).

\section{2 - PROPRIÉTÉS PHYSIQUES DES SÉDIMENTS DE LA RETENUE ET COMPORTEMENT SOUS LES ACTIONS HYDRODYNAMIQUES}

\subsection{1 - Vitesse de chute}

La vitesse de chute $W$ des sables, en eau calme, est donnée approximativement par la formule:

$$
W=120 d \quad(W \text { en } \mathrm{cm} / \mathrm{s} \text { et } d \text { en } \mathrm{cm})
$$

où $d$ est le diamètre des grains.

Les vases sont plus complexes et leurs vitesses de chute dépendent de leur concentration dans les eaux du Hamiz. A l'état de particule élémentaire, la vase aura une vitesse de chute de $0,001 \mathrm{~mm} / \mathrm{s}$ et pourra atteindre $0,13 \mathrm{~mm} / \mathrm{s}$ pour une concentration en particules solides de $20 \mathrm{~g} / 1$ (fig. 9). Au-delà de cette concentration, la chute est entravée et la vitesse diminue.

\subsection{2 * Tassement}

Les vases pures, dépourvues d'éléments supérieurs à 40 microns, se tassent au cours du temps, leur concentration moyenne passant de $350 \mathrm{~g} / 1$ après quelques jours à près de $700 \mathrm{~g} / 1$ après un an, avec un gradient entre la surface et le fond où l'on peut trouver des vases ayant une concentration de $1000 \mathrm{~g} / 1$ (densité 1,6). Exposées à l'air, ces vases subissent une dessication et peuvent atteindre $1400 \mathrm{~g} / \mathrm{l}$. En fonction de l'âge des dépôts et des conditions d'exposition à l'air, on pourra donc avoir une multitude de caractéristiques des vases.

\subsection{3 - Rhéologie}

La rigidité de la vase du Hamiz, définie par la mesure du couple nécessaire pour provoquer le début d'entrainement d'un rotor immergé dans la mixture, permet de caractériser l'état physique des dépôts (fig. 10).

Cette rigidité $\left(\tau_{y}\right.$, en $\left.\mathrm{N} / \mathrm{m}^{2}\right)$ varie rapidement avec la concentration $\left(T_{\sharp}\right)$ en particules solides. Pratiquement, pour les vases du Hamiz,

$$
\tau_{y} \neq \neq \neq n T_{s}^{4}
$$

$T_{s}$ est la masse de sédiments secs contenus dans la mixture (en $\mathrm{kg} / \mathrm{m}^{3}$ ou en $\mathrm{g} / \mathrm{l}$ )

$n$ est un facteur qui dépend du complexe sol-eau et varie entre $3,6 \cdot 10^{-11}$ pour une vase parfaitement pure et $0,8 \cdot 10^{-11}$ si cette vase contient des éléments sableux $\tau_{y}$ étant exprimé en $N / \mathrm{m}^{2}$ :

$$
\text { (1 newton } / \mathrm{m}^{2}=10 \text { dynes } / \mathrm{cm}^{2} \text { ). }
$$

Pour fixer les ordres de grandeur, une vase du Hamiz pure ayant une concentration de $500 \mathrm{~g} / 1$ aura une rigidité initiale comprise entre 0,5 et $2 \mathrm{~N} / \mathrm{m}^{2}$. A $1000 \mathrm{~g} / 1$ cette même vase aurait une rigidité qui varierait entre 20 et $80 \mathrm{~N} / \mathrm{m}^{2}$.

L'introduction de sable dans les vases modifie la rigidité initiale dans des proportions importantes (fig. 11) si l'on inclut le poids de sable dans la concentration en particules solides de la mixture. Par contre, si l'on ne considère que la teneur en eau de la vase, l'influence du sable est jugée peu importante jusqu'à $30 \%$ de sable.

La viscosité de ces vases augmente également très rapidement en fonction de la concentration, mais avec des lois plus complexes que la rigidité (fig. 12). A $500 \mathrm{~g} / \mathrm{l}$, la viscosité atteindrait 100 centipoises et seulement 5 centipoises à $100 \mathrm{~g} / 1$.

Précisons que, suivant la concentration en particules solides, la mixture pourra se comporter soit comme un fluide newtonien (faibles concentrations), soit comme un fluide de Bingham ou de Casson avec des hystérésis. Dans ce dernier cas on ne peut plus parler de viscosité absolue de la vase, les valeurs ne donnant que des éléments de comparaison entre les mixtures.

(3) Ces vases sont constituées de $40 \%$ d'illite, $25 \%$ de chlorite $15 \%$ de kaolinite et $20 \%$ d'inter stratifiés illite-kaolinite. 


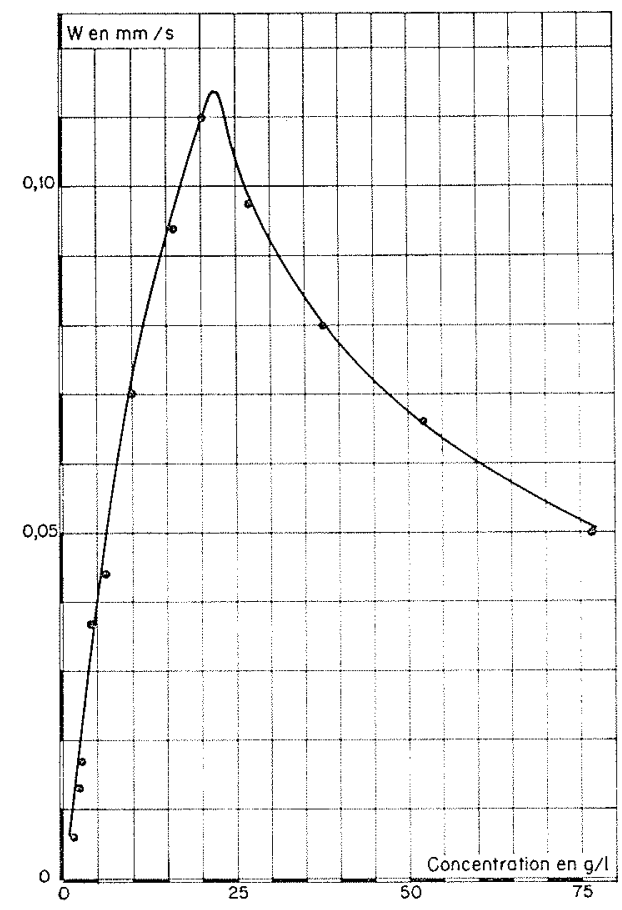

9/ Variation de la vitesse de chute des vases en fonction de la concentration (milieu aqueux naturel).

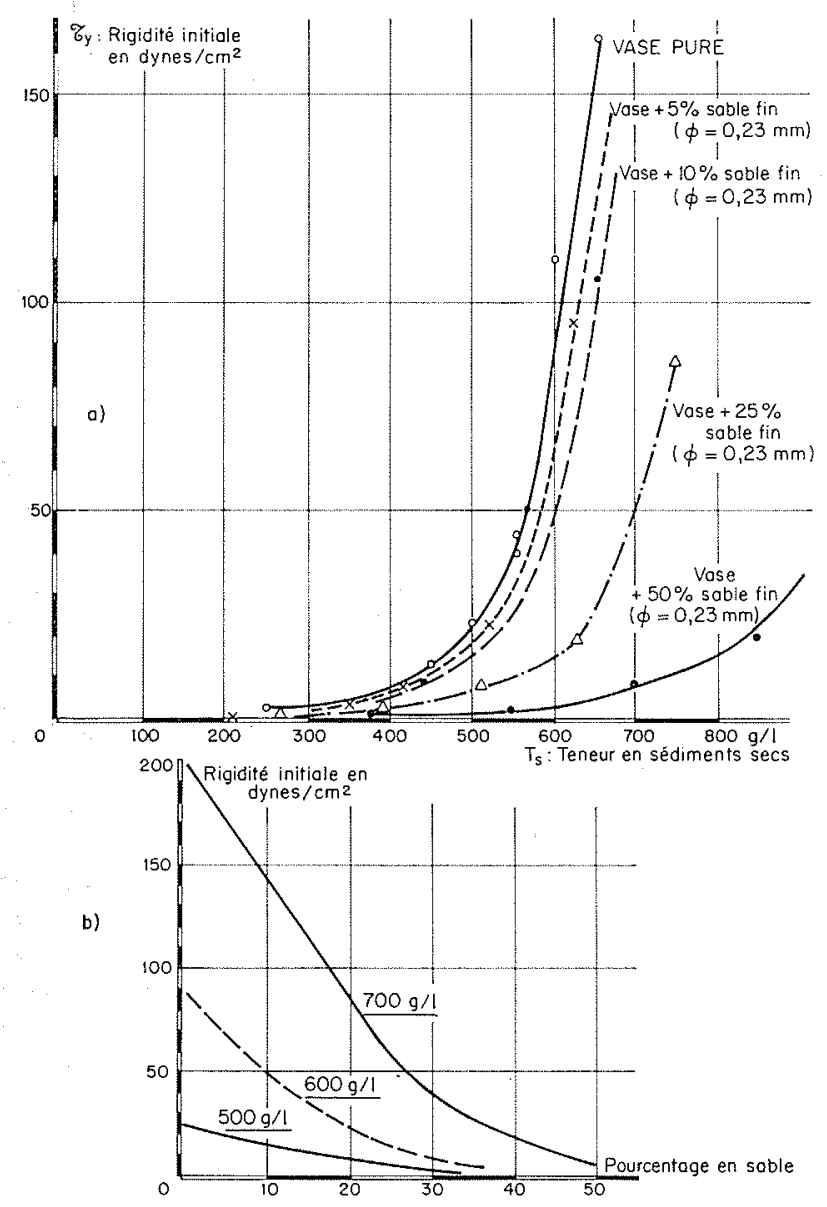

11/ Influence de la teneur en sable sur la rigidité iniliale.

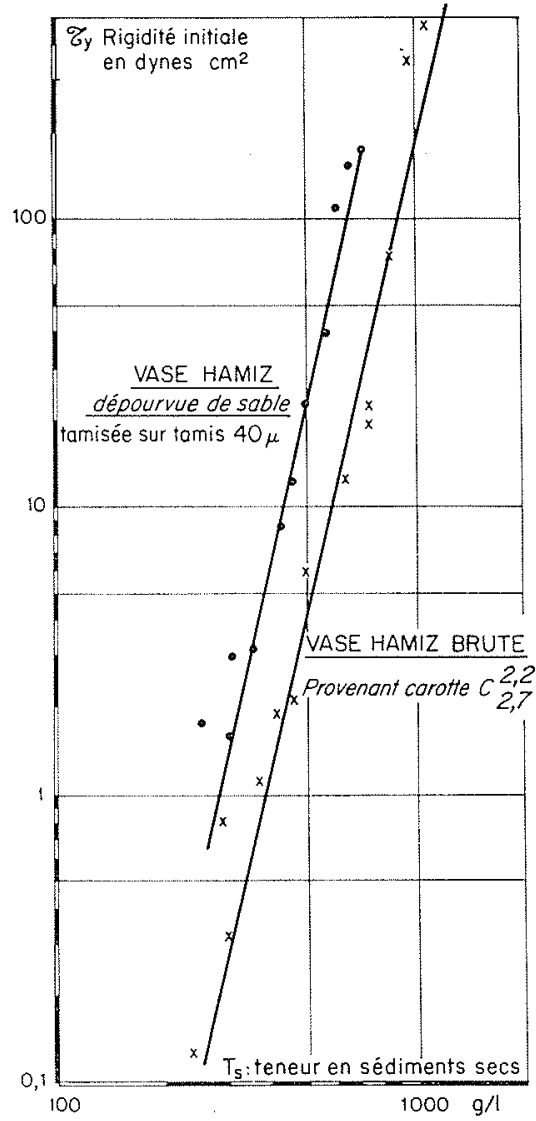

10/ Variation de la rigidité initiale des vases en fonction de la teneur en sédiment sec.

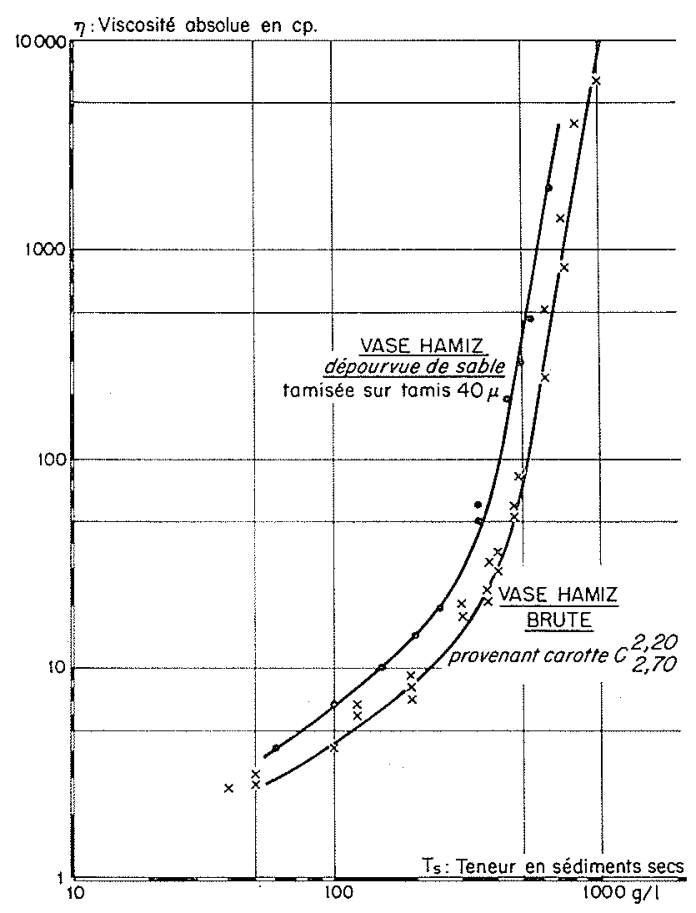

12/ Variation de la viscosité dynamique en fonction de la teneur en sédiment sec. 

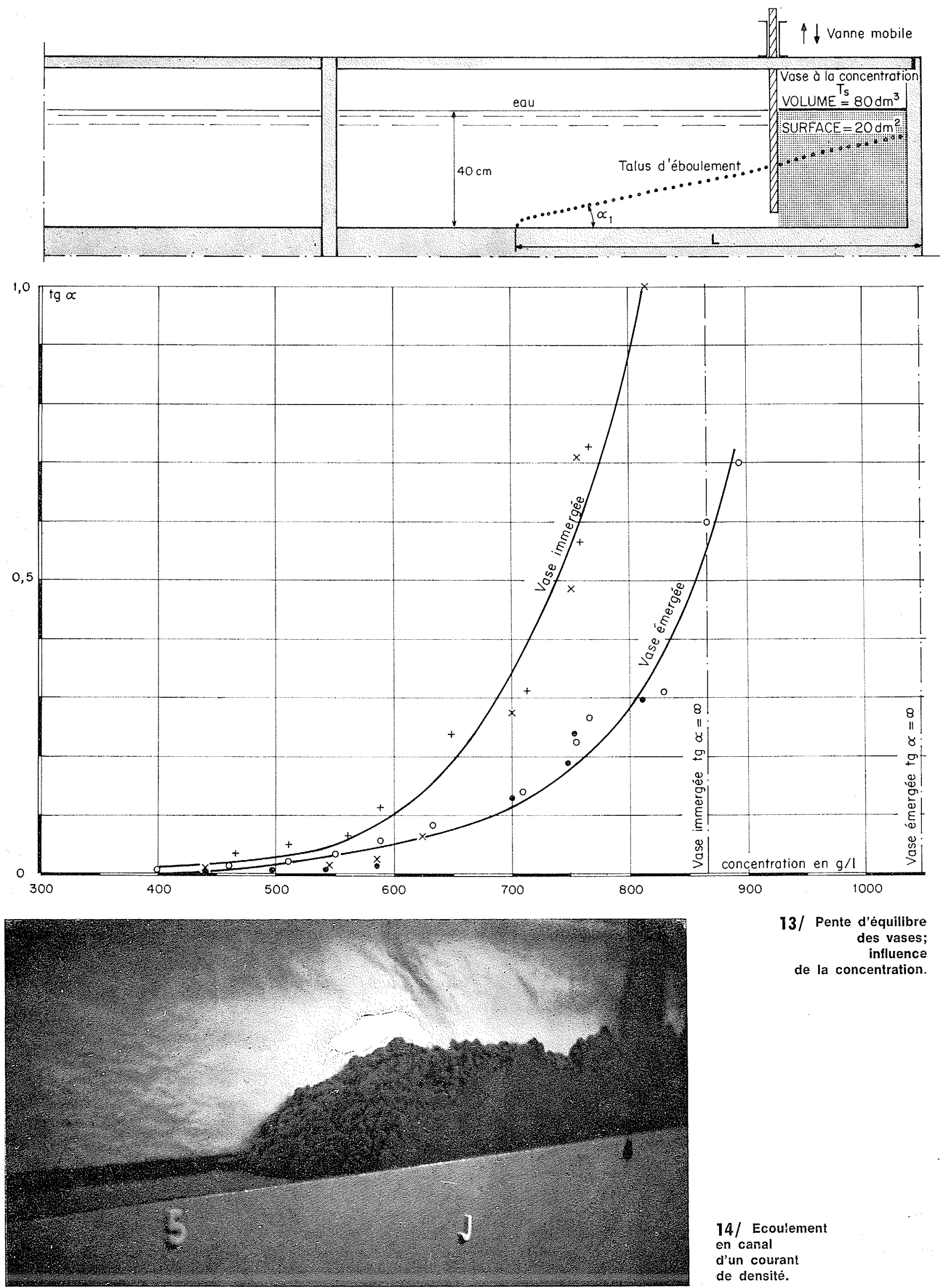

13/ Pente d'équilibre des vases; de la concentration.

14/ Ecoulement en canal

d'un courant de densité. 


\section{4 - PENTES D'ÉQUILIBRE DES DÉPOTS COURANTS DE TURBIDITÉ}

Contrairement aux sables et aux graviers qui ont des pentes d'équilibre à peu près constantes, variant en eau calme entre 30 et $40^{\circ}$ par exemple, les vases du Hamiz ont des pentes très variables suivant leur état de tassement.

D'une façon générale, la pente des dépôts, caractérisée par la tangente de l'angle à la base $(\operatorname{tg} \alpha)$, dépendra directement de la rigidité initiale $\tau_{y}$ et sera différente suivant que le sol est immergé ou émergé (fig. 13).

$$
\begin{aligned}
& \text { Vase inmergée } \ldots \operatorname{tg} \alpha=0,022 \tau_{y \prime}\left(\text { en } \mathrm{N} / \mathrm{m}^{2}\right) \\
& \text { Vase émergée } \ldots \\
& \quad \operatorname{tg} \alpha^{\prime}=0,01 \tau_{y}\left(\text { en } \mathrm{N} / \mathrm{m}^{2}\right)
\end{aligned}
$$

La présence de sable modifiera assez peu la pente des dépôts tant que le pourcentage de sable ne dépassera pas $30 \%$ dans une vase de concentration déterminée en éléments pélitiques.

Notons que si la concentration de la mixture est faible les vases pourront s'écouler au voisinage du fond sous forme d'un courant de densité et se propager très loin de la retenue (fig. 14) en abandonnant d'ailleurs une partie des éléments sableux qu'elles contiennent.

\section{5 - EROSION ET TRANSPORT DES SÉDIMENTS}

Rappelons qu'un sédiment pourra être remis en mouvement si la force tractrice exercée par le fluide sur le fond $\tau_{0}$ est supérieure à la force tractrice critique d'érosion du matériau $\tau_{c}$.

Rappelons également la définition de la vitesse de frottement :

$$
H_{*}=\left(\tau_{0} / \rho\right)^{1 / 2}
$$

qui peut être déterminée à partir de la répartition des vitesses $u(y), y$ étant la hauteur au-dessus du fond, connaissant le coefficient de rugosité $k_{s}$.

Pour les sédiments du Hamiz, de nombreuses mesures en canal ont permis de déterminer les forces tractrices critiques pour différentes caractéristiques du matériau. On constate que dans ce cas:

- les sables sont remis en mouvement dès que la force tractrice (en $\mathrm{N} / \mathrm{m}^{2}$ ) est supérieure à 0,9 fois le diamètre des grains (en $\mathrm{mm}$ ):

$$
\tau_{0}\left(\mathrm{~N} / \mathrm{m}^{2}\right) \geqslant 0,9 d(\mathrm{~mm})
$$

- les vases pures sont érodées et remises en suspension dès que la force tractrice est supérieure ou égale à la rigidité initiale du dépôt $\tau_{y}$.

$$
\tau_{0} \geqslant \tau_{y}
$$

En fait, les vases du Hamiz contenant des éléments silteux ou sableux, l'érosion des dépôts pourra s'effectuer pour des forces tractrices sensiblement inférieures à la rigidité initiale mesurée sur des vases pures. La figure 15 donne les vitesses critiques de frottement $u *$ nécessaires pour éroder une vase prélevée dans la retenue du barrage et ayant différentes concentrations.
Notons également que les vases du Hamiz (comme celles de l'Oued Fodda) sont plus facilement érodées, à concentration égale, que des vases marines par exemple. Ce fait est dû à la composition minéralogique propre des vases du Hamiz, qui contiennent principalement de l'illite alors que certaines vases marines sont à dominante kaolinitique.

\section{3 - Caractéristiques de l'Oued Hamiz en aval du barrage}

Les matériaux dans la retenue, dont une description sommaire vient d'être donnée, devront, une fois dragués et rejetés à l'aval du barrage, parcourir une distance de plus de $35 \mathrm{~km}$ pour atteindre la mer. La connaissance des caractéristiques de l'oued dans cette zone est nécessaire pour aborder le calcul des possibilités de transport des sédiments dans les différents tronçons.

\section{1 - PROFIL EN LONG}

La différence de niveau entre le barrage et la mer atteint $130 \mathrm{~m}$, donnant une pente moyenne de 3,5 pour mille.

Cette pente décroît graducllement de l'amont vers l'aval, passant de $6,5 \cdot 10^{-3}$ surr les 13 premiers kilomètres à $2,8 \cdot 10^{-3}$ sur les 7 kilomètres suivants et $1,1 \cdot 10^{-3}$ sur le tronçon terminal (fig. 16 et 17).

\section{2 - PROFIL EN TRAVERS ET MORPHOLOGIE DES DIFFÉRENTS SECTEURS}

En associant aux pentes de l'Oued Hamiz ses caractéristiques morphologiques, on peut en fait distinguer quatre secteurs où les problèmes de transport des matériaux peuvent être différents (fig. 18):

- Du barrage à Fondouk, l'oued présente un lit majeur de 10 à $20 \mathrm{~m}$ de largeur entaillé dans des dépôts anciens (fig. 19); la vallée s'élargit ensuite progressivement vers l'aval (fig. 20). Les fonds sont tapissés d'éléments à granulométrie dispersée contenant des graviers et de gros galets.

- De Fondouk à Hamedi, bien que la pente du profil en long reste forte $\left(5.10^{-3}\right)$, le lit de l'oued s'élargit considérablement et peut atteindre $100 \mathrm{~m}$ de largeur. On ne retrouve pas de traces de creusement dans d'anciens dépôts et les fonds sont pavés de gros éléments apportés et déposés dans ce secteur au cours des crues (fig. 21). Il faut atteindre le confluent de l'Oued Hamiz et de 1'Oued Barek pour que des dépôts de sable apparaissent.

- De Hamedi à la route nationale 5, c'est-à-dire à $25 \mathrm{~km}$ environ en aval du barrage, le profil de l'oued devient très calibré à l'intérieur des terrains limoneux de la Mitidja et la pente d'écoulement diminue considérablement $\left(1\right.$ à $\left.1,5 \cdot 10^{-3}\right)$. La largeur du lit majeur est voisine de $20 \mathrm{~m}$ avec une profondeur de $5 \mathrm{~m}$ environ et de nombreux méandres apparaissent (fig. 22). Les galets et graviers sont de plus en plus rares et les sables fins, mélangés quelquefois à des limons, apparaissent. 

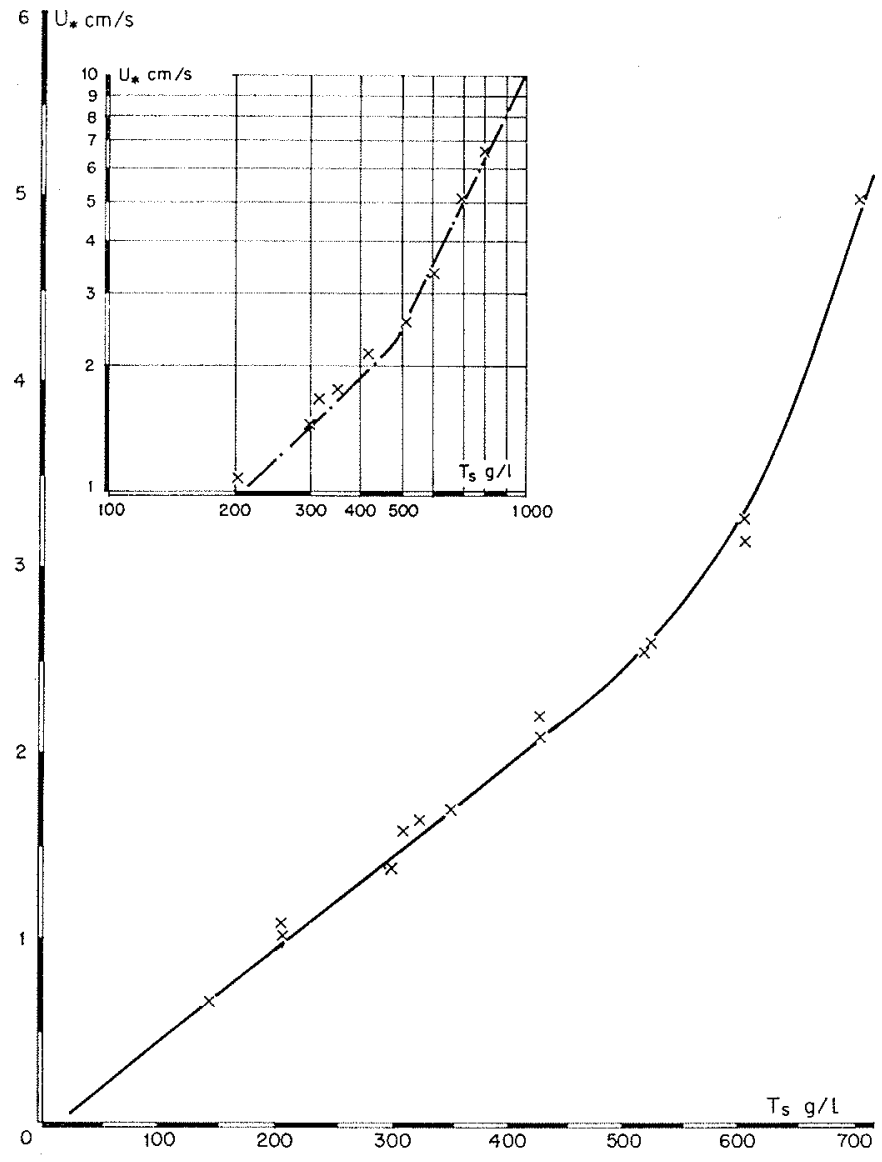

15/ Vitesse critique de frottement des vases du Hamiz; influence de la teneur en sédiment sec.
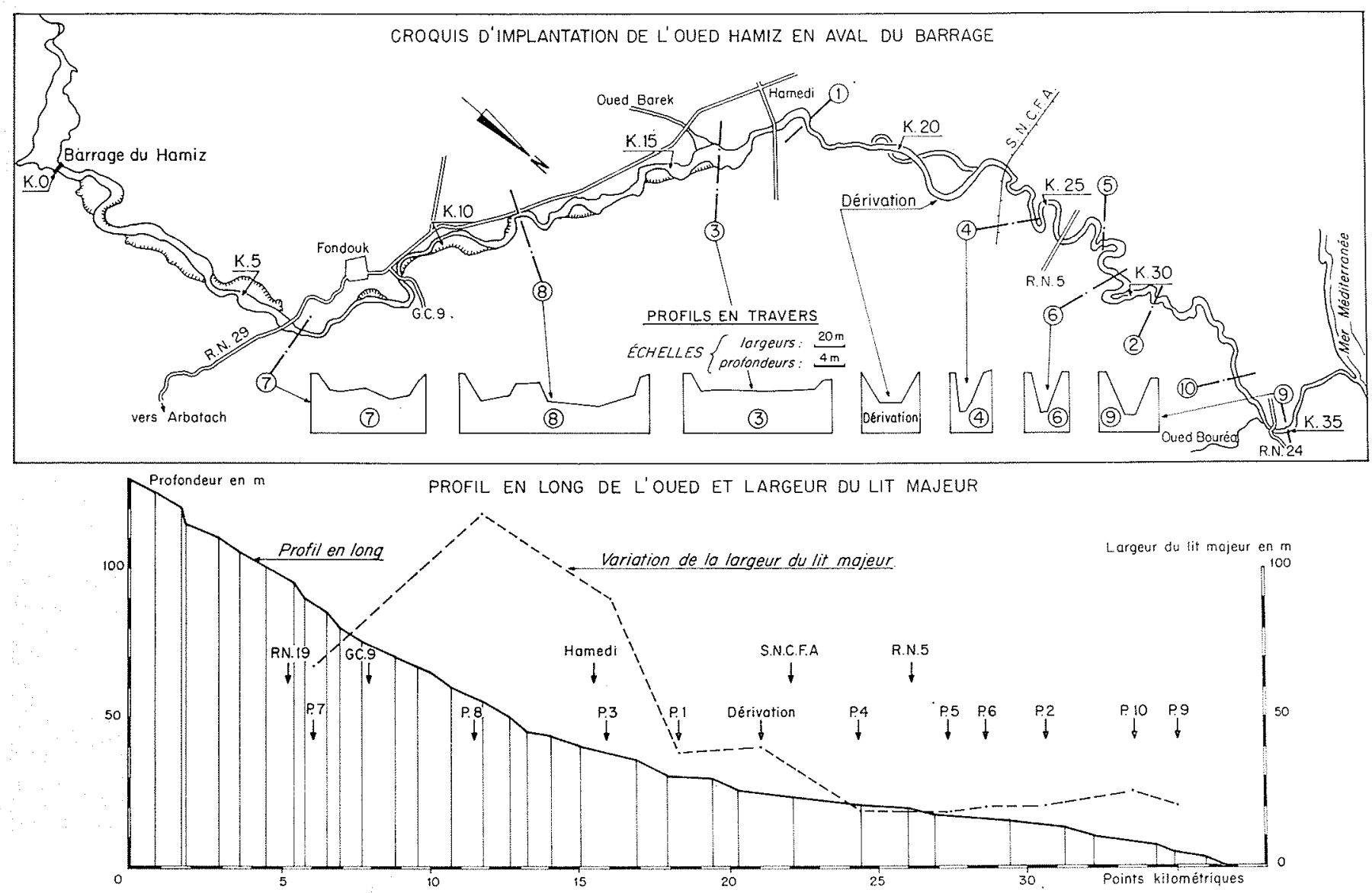


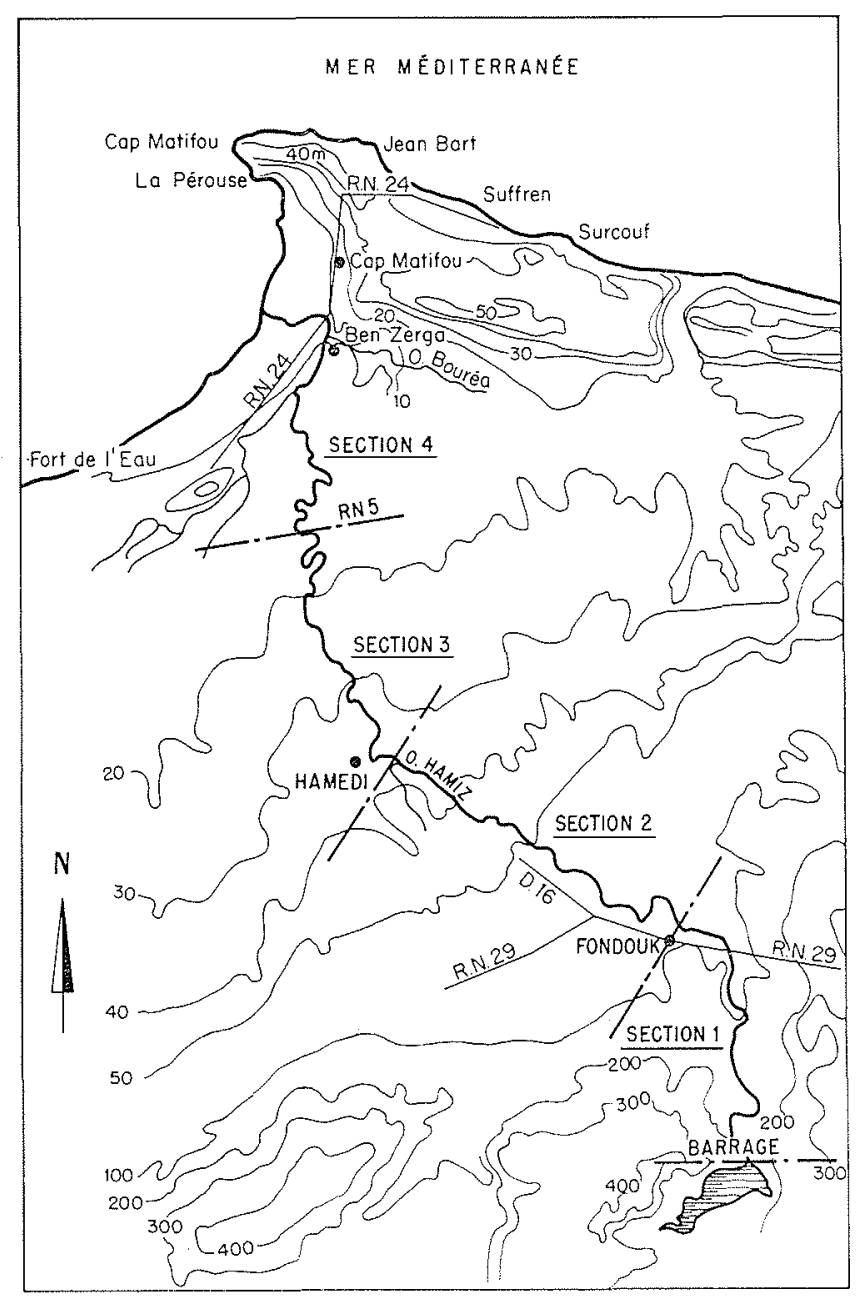

$18 /$

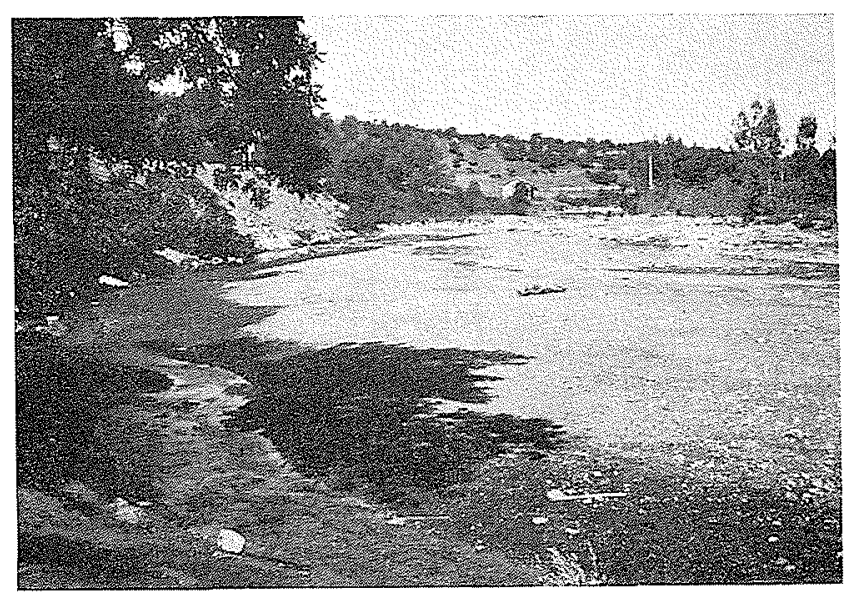

20/ Section 1.

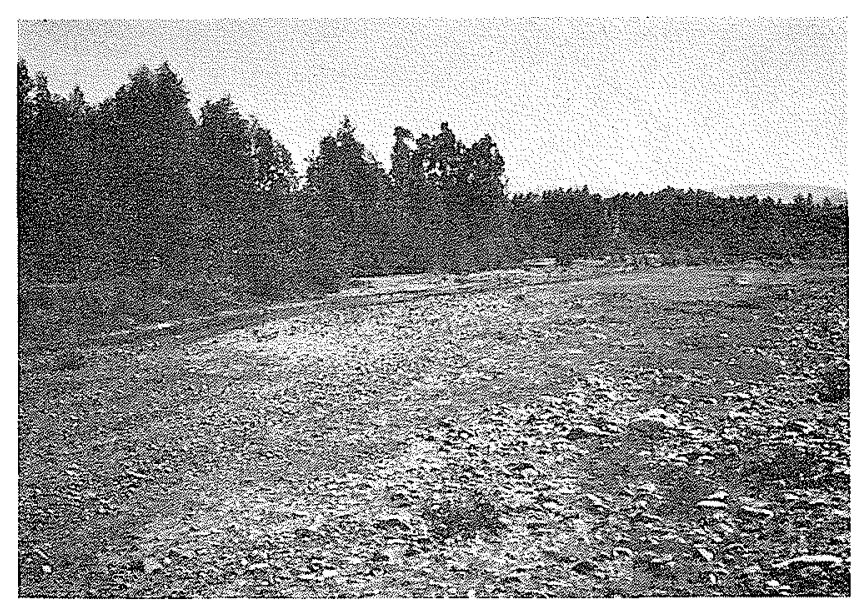

2I/ Section 2.

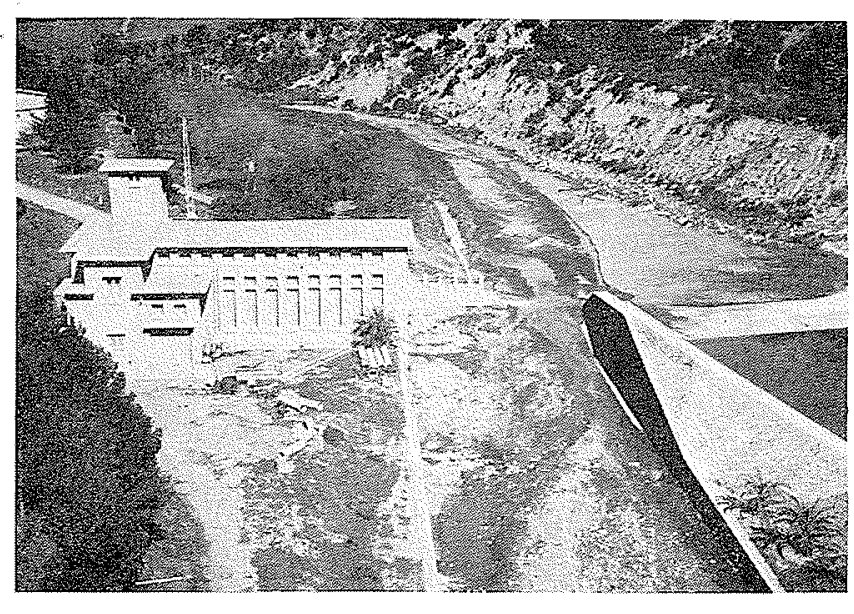

19/ Section 1.

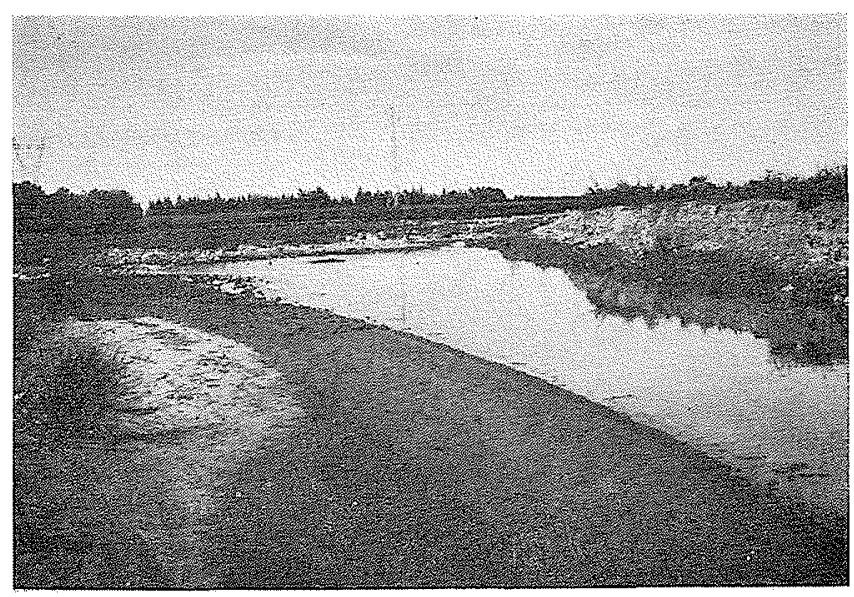

22/ Section 3. 
- De la route nationale à la mer, le profil de l'oued reste très encaissé, les fonds sont constitués uniquement d'éléments fins et les berges couvertes d'une végétation intense, la pente des fonds atteint $2,4 \cdot 10^{-3}$ après le confluent avec l'Oued Bourea.

Pour chacun de ces secteurs, les sections et périmètres mouillés et les rayons hydrauliques ont été définis en fonction de la hauteur d'eau permettant de réunir les éléments indispensables aux calculs hydrauliques et sédimentologiques.

\section{3 - ÉTUdE DES DÉPOTS SÉDIMENTAIRES APRẼS DIFFÉRENTS DÉBITS DE L'OUED ET CHASSES DU BARRAGE}

Avant d'aborder le problème de transport des sédiments dans l'Oued Hamiz en aval du barrage d'une façon théorique, on a recherché, en s'appuyant sur une étude des sédiments déposés dans l'oued après différents débits, s'il ne serait pas possible d'avoir une première estimation qualitative et empirique des risques de dépôt de différents types de matériaux dans les secteurs précédemment définis.

Dans ce but, le SEGGTH a fait procéder à de très nombreuses analyses granulométriques sur des échantillons prélevés dans l'oued après différentes chasses et crues.

Avant les chasses, les profils granulométriques montrent que les sédiments étaient assez grossiers et mal classés sur une distance de $16 \mathrm{~km}$ en aval du barrage (fig. 23). Les particules recueillies ont en général des diamètres supérieurs à $1 \mathrm{~mm}$. En aval du $\mathrm{km} \mathrm{16}$, c'est-à-dire de Hamedi, les sédiments sont beaucoup plus fins et mieux triés.

Pour un débit de $4 \mathrm{~m}^{3} / \mathrm{s}$ avec une forte concentration des eaux, variant de $180 \mathrm{~g} / \mathrm{l}$ à $6 \mathrm{~g} / \mathrm{l}$ et provenant du vidage de la retenue (évacuation de $70000 \mathrm{~m}^{3}$ ), l'Oued Hamiz ne peut transporter, au-delà du point kilométrique 20, que les vases. Les sables fins et moyens se déposent au cours des dix premiers kilomètres (fig. 24) et l'on constate que la concentration en particules solides dans les eaux décroît progressivement. Les sables recouvrent les anciens dépôts grossiers (fig. 25).

Avec un débit de $45 \mathrm{~m}^{\prime} / \mathrm{s}$ réalisé pendant une quinzaine d'heures (évacuation de 2 millions de $\mathrm{m}^{3}$ d'eau), avec une concentration moyenne en particules solides de 100 à $150 \mathrm{~g} / \mathrm{l}$, les galets et graviers se déposent sur les deux premiers kilomètres, les sables de 0,15 à $0,40 \mathrm{~mm}$ sur les 8 kilomètres suivants et les sables très fins $(0,08 \mathrm{~mm})$, mélangés à $30 \%$ de vase, se décantent au confluent avec l'Oued Barek à $16 \mathrm{~km}$ du barrage. Le triage granulométrique des sédiments de l'amont vers l'aval est très caractéristique (fig. 26).

Une crue violente de $250 \mathrm{~m}^{3} / \mathrm{s}$ pendant quelques heures et un débit de $60 \mathrm{~m}^{3} / \mathrm{s}$ pendant 24 heures, avec une concentration en particules solides ne dépassant pas 20 à $30 \mathrm{~g} / \mathrm{l}$, permet de remettre en suspension les sédiments sableux entre le barrage et le point kilométrique 18 (fig. 27). Par contre, pour ce débit la capacité de l'oued n'est pas suffisante pour transporter les galets de vase entre Fondouk et Hamedi, ni pour entraîner les sables de 0,15 à $0,5 \mathrm{~mm}$ qui se déposent en amont de la route nationale 5 . Les vases et les particules très fines peuvent être entraînées jusqu'à la mer.

Un débit de $2,6 \mathrm{~m}^{3} / \mathrm{s}$ d'eaux claires turbinées par l'usine du Hamiz ne provoque aucune remise en suspension jusqu'à Hamedi où les eaux restent parfaitement propres. Entre Hamedi et la route nationale 5 , les eaux se chargent légèrement sans que la concentration dépasse $5 \mathrm{~g} / 1$.

Ces constatations, basées uniquement sur des analyses de sédiments en place après différents régimes hydrauliques (fig. 28), permettent d'attirer l'attention sur:

- le danger de voir les sédiments grossiers (galets et graviers) se déposer immédiatement en aval du barrage et de retrouver des galets de vase en grande proportion sur les 15 premiers kilomètres;

- le risque de voir, au cours des dragages, les sables d'une granulométrie supérieure à 100 microns se déposer en presque totalité avant le pont sur la route nationale 5 , le transport jusqu'à cette zone ne s'effectuant que pour des débits importants de l'oued;

- le risque d'avoir des dépôts de sable, mélangés à une proportion plus ou moins grande de vase, sur une grande partie du cours de l'oued pour des débits inférieurs à $4 \mathrm{~m}^{3} / \mathrm{s}$, avec une forte concentration en particules solides;

- la possibilité de transporter beaucoup plus facilement les vases, même à forte concentration, que des sables fins.

\section{4 - Etude théorique de l'évacuation jusqu'à la mer des vases, des sables et des galets de vase}

En examinant le problème du transport des sédiments non plus d'une façon empirique, mais d'une façon théorique, il est possible de préciser les conditions d'évacuation jusqu'à la mer d'un volume de sédiments en fonction des débits liquides disponibles.

En ce qui concerne l'évacuation des vases, les formules usuelles ne permettent pas de résoudre ce problème. Nous avons essayé d'obtenir un ordre de grandeur des débits évacuables, pour diverses valeurs de la concentration, par une méthode théorique faisant appel à d'importantes simplifications.

Le transport par charriage des galets de vase et celui des sables en charriage et en suspension sont calculables par les méthodes classiques. L'étude de la modification du transport des sables lorsquil a lieu, non plus dans l'eau claire, mais dans une vase en suspension de caractéristiques rhéologiques très différentes, a été abordée ici par une méthode dont seule l'expérimentation pourra donner la valeur. 


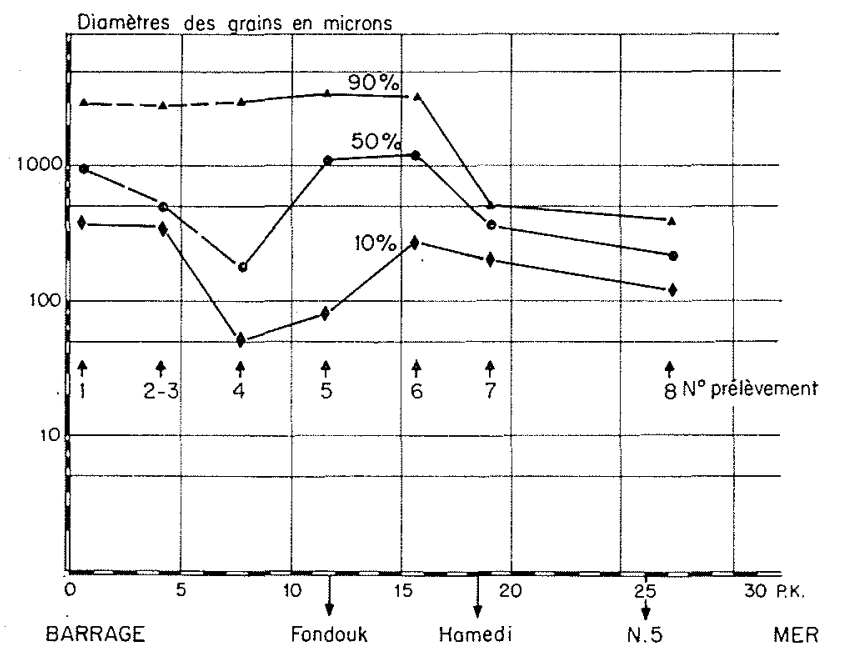

23/ Granulométrie du fond avant les chasses.

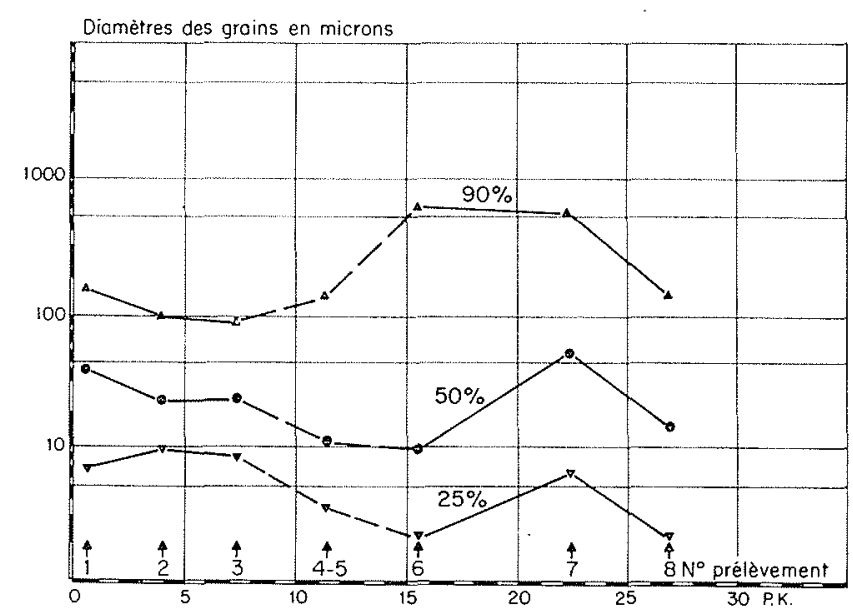

24/ Granulométrie du fond après un dëbit de $4 \mathrm{~m}^{3} / \mathrm{s}$ avec une forte concentration en sédiment.
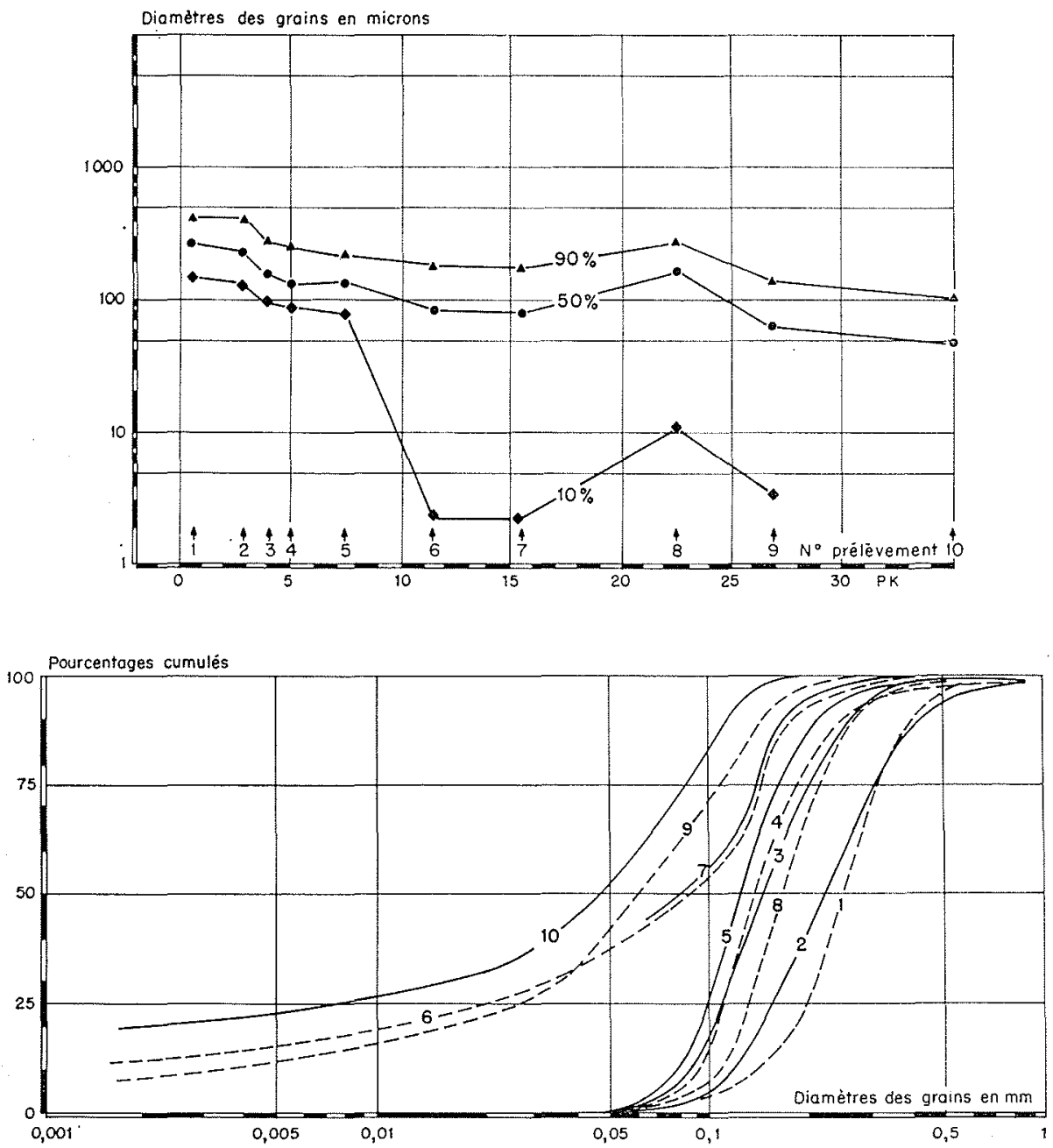

26/ Granulomètrie du fond après un débit de $45 \mathrm{~m} 3 / \mathrm{s}$ avec une concentration de 100 à $150 \mathrm{~kg} / \mathrm{m}^{3}$. 


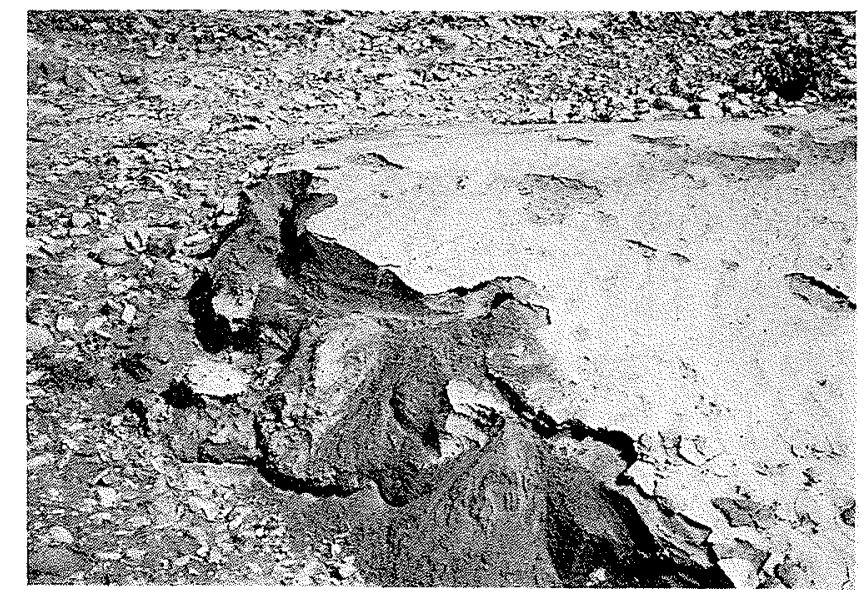

25/ Sable recouvrant d'anciens dépôts grossiers.

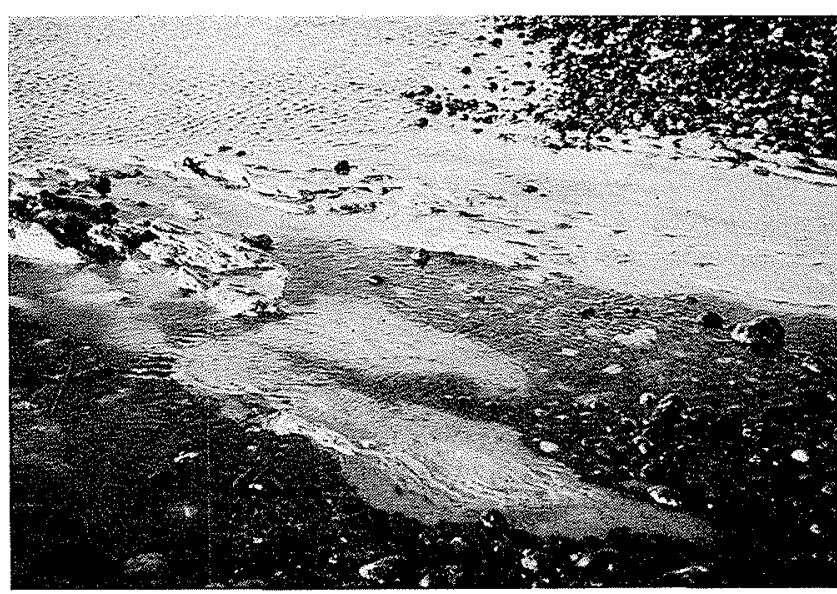

27/

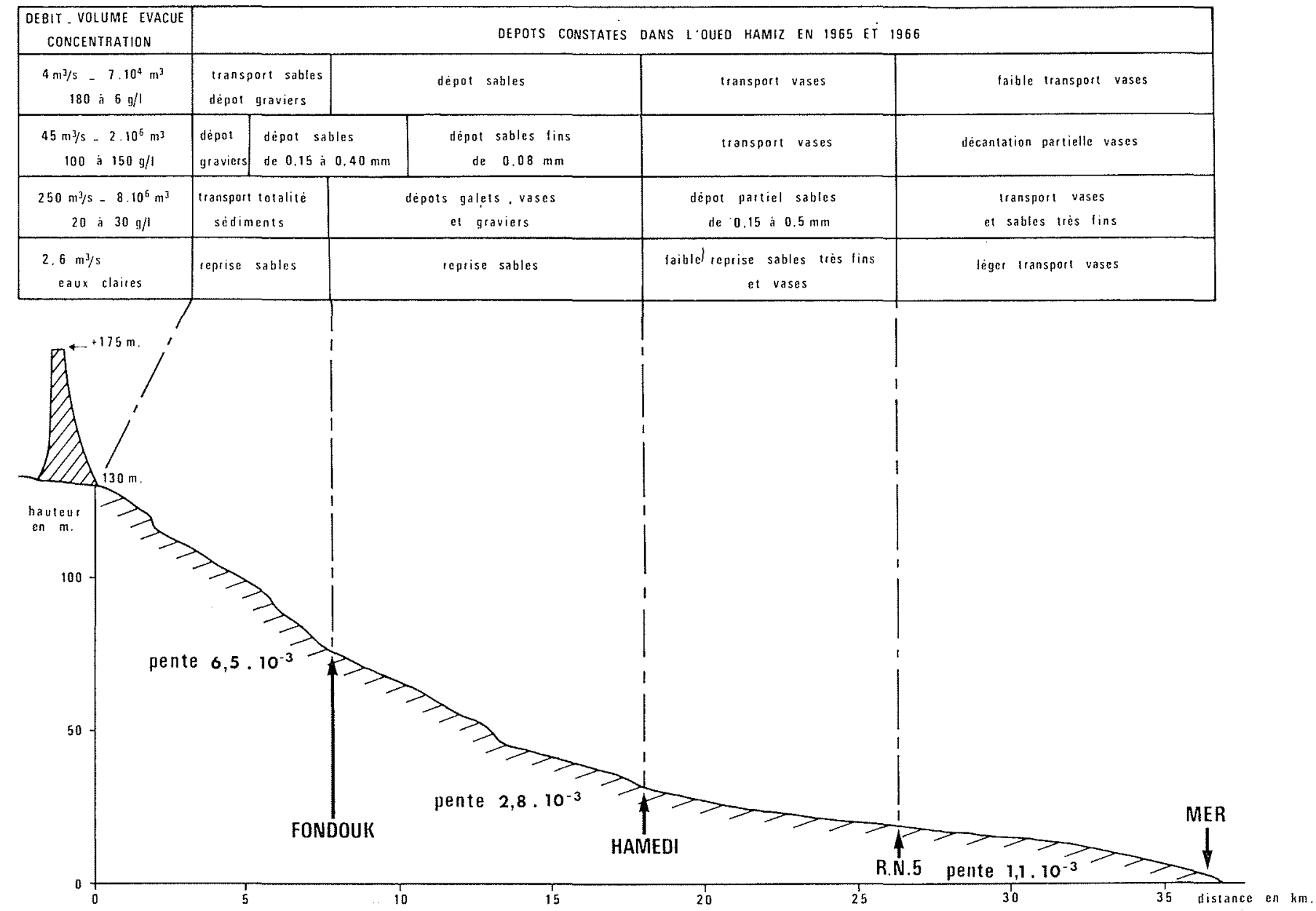




\section{1 - ÉVACUATION DES VASES}

Une vase en suspension s'écoule, lorsque les conditions requises sont remplies, comme un fluide homogène.

Pour cela, il faut que les particules suivent parfaitement le mouvement de l'eau, ce qui implique, d'après la mécanique des suspensions, la condition suivante:

$$
D u * / \nu \ll 2\left(\rho / \rho_{s}\right)^{1 / 2}
$$

- $D$ étant le diamètre de la particule;

- $u_{*}=\left(\tau_{0} / \rho\right)^{1 / 2}$ la vitesse de frottement de l'écoulement :

$\tau_{0}=$ tension tangentielle à la paroi;

$\rho=$ masse volumique de l'eau;

- $\rho_{s}$ est la masse volumique des sédiments, en général de l'ordre de 2,60 .

Si l'on prend comme ordre de grandeur $u_{*}=0,10 \mathrm{~m} / \mathrm{s}$ et $\nu=10^{-6} \mathrm{~m}^{2} / \mathrm{s}$, on obtient $D \ll 0,012 \mathrm{~mm}$. Les vases du Hamiz, dont le diamètre est de l'ordre de $0,002 \mathrm{~mm}$, satisfont donc à la relation (1).

Par ailleurs, pour que la vase reste en suspension, l'écoulement doit être turbulent. Les conditions nécessaires sont déterminées ci-dessous, dans l'hypothèse où l'on peut assimiler la suspension à un fluide de Bingham, ce qui semble être acceptable dans le cas de la vase du Hamiz.

\subsection{1 - Écoulement laminaire à surface libre d'un fluide de Bingham}

Un fluide de Bingham est caractérisé par la relation suivante entre gradient de vitesse et tension tangentielle:

$$
\eta(\partial u / \partial y)=\tau-\tau_{y}
$$

$\eta$ et $\tau_{y y}$ sont les caractéristiques rhéologiques du fiuide;

$\eta \quad$ est la viscosité dynamique ( $\nu=\eta / \rho_{m}$ étant la viscosité cinématique);

$\tau_{y} \quad$ la rigidité initiale (yield-value), en dessous de laquelle il n'y a pas d'écoulement.

Beaucoup de vases satisfont approximativement à la relation (2), $\eta$ et $\tau_{y}$ étant des fonctions de la concentration.

L'écoulement laminaire d'une telle vase dans un canal très large (à deux dimensions) de pente $i$ est schématisé sur la figure 29 (nous considérons éviđemment l'écoulement uniforme)

La tension tangentielle varie linéairement du fond à la surface. Au fond, elle est :

$$
\tau_{0}=\rho_{m} g h_{0} i
$$

$h_{0}$ étant la profondeur de l'écoulement et $\rho_{m}$ la masse volumique de la vase (mixture).

La partie supérieure, d'épaisseur $h_{t}-h_{1}=h_{0} \tau_{y /} / \tau_{0}$, se déplace en bloc, la tension tangentielle y étant plus petite que la rigidité initiale $\tau_{l y}$, donc incapable de créer un gradient de vitesse.

La partie inférieure, d'épaisseur $h_{1}$, est le siège d'un écoulement laminaire à répartition parabolique des vitesses, dû à la tension tangentielle $\tau-\tau_{y}$.

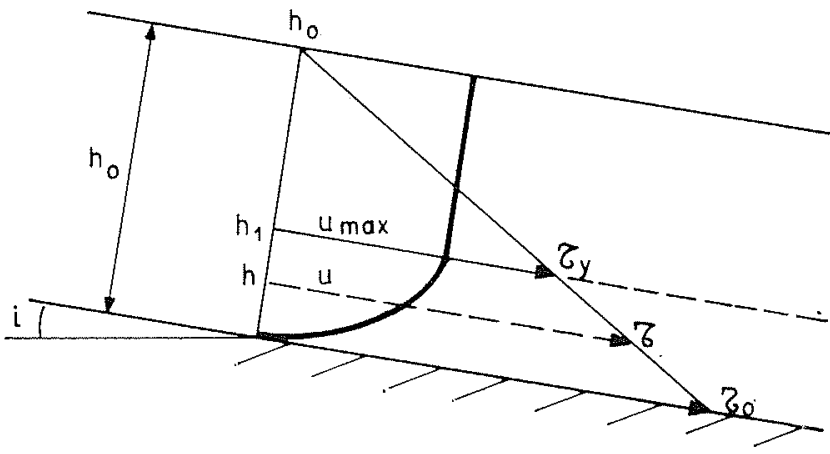

$29 /$

Le calcul en est aisé. Nous en rassemblons ci-dessous les résultats :

$$
\begin{gathered}
\tau_{0}=\rho_{m} g h i \\
h_{1}=h_{0}\left(1-\frac{\tau_{y j}}{\tau_{0}}\right) \\
u_{\mathrm{m}: \mathrm{x}}=\frac{\tau_{0} h_{0}}{2 \eta}\left(1-\frac{\tau_{y j}}{\tau_{0}}\right)^{2}
\end{gathered}
$$

de $h=0$ à $h=h_{1} \quad u=u_{\max }\left[1-\left(\frac{h_{1}-h}{h_{1}}\right)^{2}\right]$

$\operatorname{de} h=h_{1} \grave{a} h=h_{0} \quad u=u_{\max }$

Les vitesses moyennes sont:

entre $h=0$ et $h=h_{1}$ :

$$
\bar{u}_{1}=2 / 3 u_{\max }
$$

entre $h=0$ et $h=h_{0}$ :

$$
U=2 / 3 u_{\max }\left(1+0,5 \frac{\tau_{\eta}}{\tau_{0}}\right)
$$

\subsection{2 - Conditions pour que l'écoulement soit turbulent}

a) On considère une mixture caractérisée par sa teneur en sédiment $\sec T_{s}$, masse de sédiment sec contenu dans l'unité de volume de mixture $\left(\mathrm{en} \mathrm{kg} / \mathrm{m}^{3}\right)$.

La masse volumique de la mixture, $\rho_{m}$, s'en déduit, pour une masse volumique $\rho_{s}$ du sédiment de $2650 \mathrm{~kg} / \mathrm{m}^{3}$, par la relation:

$$
\rho_{m}=1000-0,615 T_{s} \quad\left(\mathrm{~kg} / \mathrm{m}^{3}\right)
$$

La rigidité initiale $\tau_{\| \prime}$ et la viscosité cinématique $\nu\left(=\eta \rho_{m}\right)$ de la mixture dépendent de $T_{s}$.

A titre d'exemple, on trouvera dans le tableau du paragraphe f) ci-après les caractéristiques des vases du Hamiz.

b) Ia mixture s'écoule, dans le cas présent, dans le lit de l'Oued Hamiz, qui présente des caractéristiques (section droite, pente, rugosité) variables avec l'emplacement de la section. 
On considérera, pour l'étude des conditions dans lesquelles l'écoulement peut rester turbulent, une zone prismatique caractérisée par une section droite rectangulaire de largeur $B$, une pente du fond $i$ et une rugosité définie par le coefficient de Strickler $K$. Dans une telle zone, l'écoulement uniforme est défini par le tirant d'eau $h_{0}$.

c) Une première relation entre le tirant d'eau et le débit par unité de largeur $q$ est donnée par la formule de Strickler :

$$
\begin{gathered}
q=K h_{0}{ }^{5 / 3} i^{1 / 2} \\
\left(q \text { en } \mathrm{m}^{2} / \mathrm{s}, \quad h_{0} \text { en } \mathrm{m}\right)
\end{gathered}
$$

Utiliser cette formule revient à faire l'hypothèse qu'on est en régime turbulent rugueux, ce que l'on peut admettre pour une rivière naturelle. Nous devrons cependant nous en souvenir.

d) Nous pouvons par ailleurs définir, pour l'écoulement de la mixture considérée dans le canal ainsi défini, un tirant d'eau minimum $h_{\min }$ en-dessous duquel il n'y aurait pas d'écoulement.

En effet, la tension tangentielle au fond est:

$$
\tau=p_{m} g h_{0} i
$$

Pour qu'il y ait écoulement, il faut qu'elle soit supérieure à $\tau_{y /}$, rigidité initiale de la mixture, donc que:

$$
h>h_{\mathrm{nin}}=\tau_{y} / p_{m} g i
$$

e) La condition de turbulence, pour un écoulement d'un fluide newtonien comme l'eau, dans un canal rectangulaire, est que le nombre de Reynolds:

$$
\mathfrak{R}=4 U h / \nu
$$

soit supérieur à une valeur critique, pour laquelle nous prendrons la valeur 2000 .

- $U$ est la vitesse moyenne dans la section;

- $\nu$ la viscosité cinématique (qui suffit à définir la rhéologie du fluide newtonien).

Pour appliquer une relation de ce genre à un fluide de Bingham, il faudrait définir une viscosité cinématique équivalente $v_{\text {and }}$ qu'on puisse y faire intervenir.

Si l'on considère l'écoulement schématisé sur la figure 29 , on peut assimiler la partie inférieure de cet écoulement, de hauteur $h_{1}$, à un écoulement laminaire d'un fluide newtonien de viscosité $\nu_{1}$, avec une tension tangentielle à la paroi égale à $\tau_{0}$.

La valeur de $\nu_{1}$ peut se déduire des relations concernant l'écoulement newtonien équivalent et l'écoulement réel :

$$
\begin{gathered}
\nu_{1} \rho_{m}(\partial u / \partial z)_{0}=\tau_{0} \\
\nu \rho_{m}(\partial u / \partial z)_{0}=\tau_{0}-\tau_{\nu}
\end{gathered}
$$

$\nu$ étant la viscosité mesurée (et qui dépend de $T_{s}$ ).

Il vient :

$$
\nu_{1}=\nu(1-\alpha)
$$

si l'on pose $\tau_{y} / \tau_{0}=h_{\mathrm{min}} / h_{0}=\alpha$.
La condition pour que l'écoulement soit turbulent est:

$$
4 \bar{u}_{1} h_{1} / \nu_{1}>2000
$$

$\bar{u}_{1}$ étant la vitesse moyenne entre le tirant d'eau 0 et le tirant d'eau $h_{1}$.

La relation que nous cherchons doit relier la vitesse moyenne $U=q / h_{0}$ au tirant d'eau total $h_{0}$. Tenant compte de (3 bis), (5), (6) et (11), la relation (12) devient:

$$
\frac{4 U h_{0}}{\nu} \frac{(1-\alpha)^{2}}{(1+0,5 \alpha)}>2000
$$

$U h_{0}$ est le débit par unité de largeur $q$. La relation ci-dessus revient à définir un débit minimum $q_{\mathrm{min}}$ en dessous duquel l'écoulement ne peut être turbulent:

$$
q_{\min }=500 \nu \frac{(1+0,5 \alpha)}{(1-\alpha)^{2}}
$$

Dans cette relation, $q_{\min }$ est une fonction de $T_{s}$ (par $\nu$ et $\left.\alpha=h_{\min } / h=\tau_{y} / \rho_{n} g h_{0} i\right)$ et de $h$.

Pour une valeur donnée de $T_{s}$, nous avons donc deux relations, (8) et (13), reliant $q$ et $h_{0}$, ce qui nous permet de déterminer facilement le tirant d'eau et le débit au-dessus desquels il faut se placer pour que la mixture s'écoule en régime turbulent

On peut simplifier les calculs, étant donné la faible précision requise, en remplaçant la relation (13) par la relation (13 bis), qui en donne une approximation suffisante pour $h_{0} / h_{\min }$ compris entre 2 et 7 :

$$
q_{\min }=5000 \vee h_{\min } / h_{0}
$$

Le calcul du tirant d'eau $h$ et du débit par unité de largeur $q$, permettant d'évacuer la mixture, se font alors au moyen des relations ci-dessous :

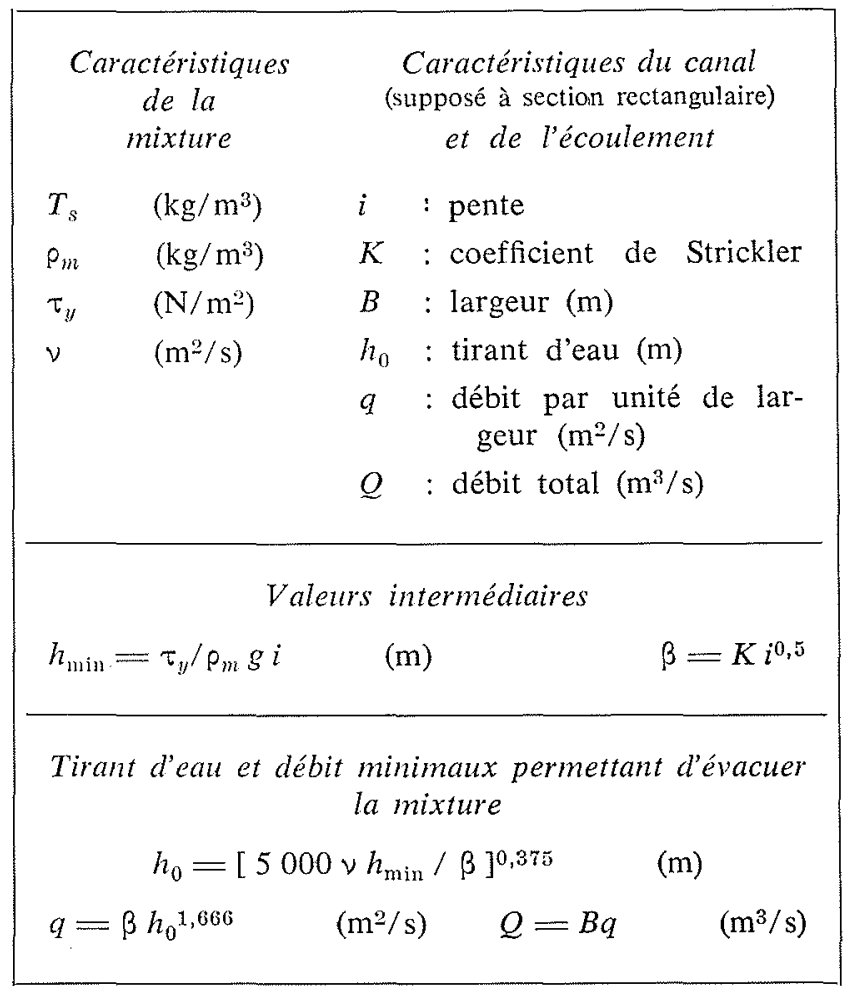


On pourra vérifier sur l'exemple ci-après que les résultats fournis par ces relations sont très voisins de ceux obtenus en utilisant (8) et (13).

h) Exemple: Ce calcul est appliqué à la zone la plus critique du Hamiz (entre Hamedi et la route nationale $n^{\circ} 5$ ). La section est assimilée à une section rectangulaire ayant les caractéristiques suivantes:

$$
i=6.10^{-4} \quad B=40 \mathrm{~m} \quad K=40
$$

Il donne les résultats regroupés ci-dessous, $Q$ étant le débit total de mixture $(Q=B q)$, et $M$ le débit en masse de sédiment $\sec \left(M=Q T_{s}\right)$.

\begin{tabular}{|c|c|c|c|c|}
\hline$T_{s} \ldots \ldots \ldots\left(\mathrm{kg} / \mathrm{m}^{3}\right)$ & 200 & 350 & 450 & 500 \\
\hline$\rho_{m} \ldots \ldots \ldots\left(\mathrm{kg} / \mathrm{m}^{3}\right)$ & 1123 & 1215 & 1280 & 1308 \\
\hline$\tau_{y} \ldots \ldots \ldots\left(\mathrm{N} / \mathrm{m}^{2}\right)$ & 0,06 & 0,33 & 1,25 & 2,265 \\
\hline$\nu \ldots \ldots\left(10^{-6} \mathrm{~m}^{2} / \mathrm{s}\right)$ & 13,4 & 43 & 142 & 215 \\
\hline$h_{\min } \ldots \ldots \ldots(\mathrm{m})$ & 0,01 & 0,045 & 0,16 & 0,29 \\
\hline$h_{0} \ldots \ldots \ldots \ldots(\mathrm{m})$ & 0,064 & 0,17 & 0,40 & 0,64 \\
\hline $\mathrm{Q} \ldots \ldots \ldots\left(\mathrm{m}^{3} / \mathrm{s}\right)$ & 0,40 & 1,9 & 9,1 & 18,4 \\
\hline$Q$, calcul simplifié. & 0,39 & 2,2 & 10,5 & 19,5 \\
\hline$M \ldots \ldots \ldots(\mathrm{kg} / \mathrm{s})$ & 80 & 660 & 4100 & 9200 \\
\hline
\end{tabular}

En pratique, étant donné les hypothèses faites sur la rhéologie de l'écoulement, et les simplifications inévitables dans toute théorie concernant des phénomènes naturels aussi complexes, il serait prudent de considérer ces valeurs comme des ordres de grandeur et, dans la pratique, de prendre une marge de sécurité importante, par exemple d'utiliser pour une concentration donnée un débit double de celui que donne le calcul. On obtient ainsi un ordre de grandeur des débits qui devraient être utilisés pendant les travaux de dragage pour amener les vases jusqu'à la mer.

Ces résultats expliquent également les concentrations énormes de vase $\left(500\right.$ à $\left.600 \mathrm{~kg} / \mathrm{m}^{3}\right)$ que l'on peut trouver dans les oueds algériens au moment des crues. Ils montrent que de tels matériaux peuvent être transportés assez facilement.

\section{2 - ÉVACUATION DES SABLES}

L'évaluation du transport des sables dans les différents tronçons de l'Oued Hamiz a été faite en utilisant d'une part les formules classiques d'Einstein et de Bijker, les sédiments étant supposés transportés dans de l'eau pure, d'autre part en essayant d'appliquer aux sables les résultats obtenus dans le domaine du transport des sédiments en conduite.

\subsection{1 - Transport des sables dans l'eau pure}

La méthode d'Einstein permet de faire le calcul du débit solide totale par fractions de la courbe granulométrique. On a admis dans le cas du Hamiz une granulométrie moyenne comportant $10 \%$ d'éléments supérieurs à $12 \mathrm{~mm}$, $15 \%$ compris entre 12 et $1,2 \mathrm{~mm}, 35 \%$ entre 1,2 et $0,5 \mathrm{~mm}, 30 \%$ entre 0,5 et $0,17 \mathrm{~mm}$ et $10 \%$ entre 0,17 et $0,03 \mathrm{~mm}$.

Le calcul a été effectué pour des débits compris entre 1 et $10 \mathrm{~m}^{3} / \mathrm{s}$ dans deux zones caractéristiques extrêmes situées, d'une part en aval de la route nationale 5, c'est-à-dire dans une zone où la pente minimale des fonds est de $6.10^{-4}$, d'autre part entre le barrage et Fondouk dans un secteur où la pente des fonds atteint $6,7.10^{-3}$.

Les résultats de ces calculs sont donnés dans le tableau ci-après, les vitesses indiquées étant celles déterminées par le calcul hydraulique donné par la méthode d'Einstein. On constate que, si dans la zone de forte pente le débit solide peut atteindre $150 \mathrm{~kg} / \mathrm{s}$ pour un débit de $10,5 \mathrm{~m}^{3} / \mathrm{s}$ (moins de $15 \mathrm{~g} / \mathrm{l}$ ), il ne dépasse pas $3 \mathrm{~kg} / \mathrm{s}$ pour le même débit dans la zone de faible pente.

\begin{tabular}{|c|c|c|c|c|}
\hline \multicolumn{5}{|c|}{ ZONE A FAIRLE PENTE $\left(6.10^{-7}\right)$ : } \\
\hline Débit liquide . $\left(\mathrm{m}^{3} / \mathrm{s}\right)$ & 1,95 & 4,80 & 7,85 & 10,9 \\
\hline Vitesse $\ldots \ldots(\mathrm{m} / \mathrm{s})$ & 0,46 & 0,71 & 0,90 & 1,06 \\
\hline Débit solide $\ldots(\mathrm{kg} / \mathrm{s})$ & 0 & 0,45 & 1,65 & 3,10 \\
\hline \multicolumn{5}{|c|}{ ZONE A FORTE PENTE $\left(6,7,10^{-3}\right)$ : } \\
\hline Débit liquide . $\left(\mathrm{m}^{3} / \mathrm{s}\right)$ & 1,29 & 3,31 & 6,34 & 10,5 \\
\hline Vitesse $\ldots \ldots(\mathrm{m} / \mathrm{s})$ & 1,01 & 1,57 & 2,02 & 2,42 \\
\hline Débit solide .. $(\mathrm{kg} / \mathrm{s})$ & 3 & 33 & 70 & 153 \\
\hline
\end{tabular}

Cette méthode donne le transport solide total, par charriage et en suspension.

\subsection{2 - Évaluation des transports de sable en suspension dans une mixture de vase}

Les formules utilisées plus haut ne permettent pas d'évaluer la modification du débit de sable transporté en suspension lorsque le milieu transporteur a des caractéristiques rhéologiques très différentes de celles de l'eau. L'approche ci-dessous peut donner des indications sur ce point.

Si l'on considère un écoulement donné d'eau ou de mixture vaseuse, il peut admettre en suspension un sable de diamètre donné avec une certaine concentration en poids $C_{p}$ (poids du sable/poids total) croissant avec le tirant d'eau et avec la pente.

Si l'on admet que la puissance consacrée au maintien du sable en suspension peut être une fraction donnée de la puissance perdue par l'écoulement - cette hypothèse n'est probablement pas exacte, mais elle va dans le sens des choses - on peut montrer que la concentration maximum admissible, pour un écoulement donné, est inversement proportionnelle à la vitesse de chute $W_{0}$ du sable.

Une mixture de vase est plus dense que l'eau pure et elle a une viscosité beaucoup plus forte. Par suite, la 
vitesse de chute des grains de sable y sera plus faible, et la concentration admissible plus importante.

Le sable du Hamiz, par exemple, a dans l'eau pure $\left(v=10^{-6} \mathrm{~m}^{2} / \mathrm{s}\right)$ une vitesse de chute de l'ordre de $8 \cdot 10^{-2} \mathrm{~m} / \mathrm{s}$. Dans une mixture de vase à $500 \mathrm{~kg} / \mathrm{m}^{3}$ $\left(\nu=215 \cdot 10^{-6} \mathrm{~m}^{2} / \mathrm{s}, \quad P_{m i}=1308 \mathrm{~kg} / \mathrm{m}^{3}\right)$, la vitesse de chute du même sable serait de l'ordre de $1 \mathrm{~mm} / \mathrm{s}$, soit 80 fois plus faible. La concentration admissible serait done 80 fois plus importante.

\section{3 - ÉVACUATION DES GRAVIERS, Galets ET GALETS DE VASE}

Le pourcentrage de graviers et galets de densité voisine de 2,5 à 2,6 est relativement faible dans la retenue et ne devrait pas poser de problèmes très importants au moment du dragage. Par contre, les galets de vase qui peuvent être soit naturels, comme on l'a vu au début de cet exposé, soit artificiels, et provenir des conditions de dragage dans un sol constitué de vases ayant atteint une forte rigidité, risquent d'être importants. Une telle production de galets de vase s'est trouvée au moment des dragages du barrage du Cheurfas (fig. 30) entraînant un colmatage provisoire de l'oued en aval du barrage.

\subsection{1 - Transport des galets et graviers}

La formule de Meyer-Peter rend compte des possibilités de transport dans les différents tronçons de l'Oued Hamiz.

Dans le tronçon critique (Hamedi, route nationale $n^{\circ} 5$ ), il faudrait des débits supérieurs à $100 \mathrm{~m}^{3} / \mathrm{s}$ pour transporter des graviers de $1 \mathrm{~cm}$ de diamètre et de plus de $1000 \mathrm{~m}^{3} / \mathrm{s}$ pour transporter des galets de $5 \mathrm{~cm}$ de diamètre.

Dans la zone de forte pente, entre le barrage et Fondouk, il faudrait dépasser des débits de $90 \mathrm{~m}^{3} / \mathrm{s}$ pour transporter des galets de $5 \mathrm{~cm}$ et $270 \mathrm{~m}^{3} / \mathrm{s}$ pour des galets de $10 \mathrm{~cm}$.

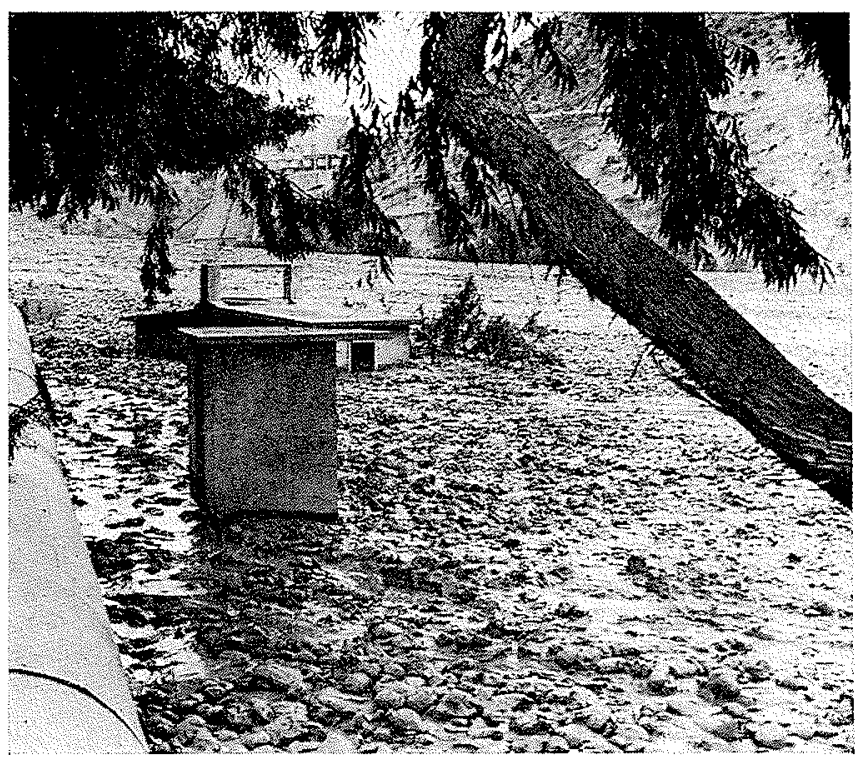

30/ Galets de vase au barrage du Cheurfas.

\subsection{2 - Transport des galets de vase}

Le calcul des possibilités de transport des galets de vase a été effectué en utilisant la formule de Bijker et en admettant que ces galets avaient une densité de 2,0 ce qui représente une très forte concentration en particules solides, et un diamètre de $10 \mathrm{~cm}$. Le coefficient de Strickler a été pris égal à 40 comme pour les autres calculs.

Le tableau ci-après donne le débit solide en galets de vase par unité de largeur du lit pour diverses valeurs du débit liquide $q$ par unité de largeur et différentes pentes du lit de l'oued.

\begin{tabular}{|c|c|c|c|c|c|}
\hline \multicolumn{6}{|c|}{ DÉBIT SOLIDE $(\mathrm{en} \mathrm{kg} / \mathrm{s}$ par m) } \\
\hline $\begin{array}{l}\text { Débit par unité de lar- } \\
\text { geur }\left(\mathrm{en}^{3} / \mathrm{s} \text { par }\right. \\
\mathrm{m})\end{array}$ & 1 & 5 & 10 & 20 & 50 \\
\hline Pentes $5.10^{-4} \ldots \ldots$ & 0 & 0 & 0 & 1 & 55 \\
\hline $10^{-3} \ldots \ldots$ & 0 & 0 & 4 & 45 & 340 \\
\hline $2.10^{-3} \ldots$ & 0 & 5 & 60 & 260 & 1100 \\
\hline $5.10^{-3}$. & 0 & 80 & 500 & 1100 & 4000 \\
\hline
\end{tabular}

Sur les sept premiers kilomètres, où la pente de l'oued dépasse $5 \cdot 10^{-3}$, il sera possible d'entraîner des galets de vase en quantité appréciable dès que le débit dépassera $100 \mathrm{~m}^{3} / \mathrm{s} ;$ avec $200 \mathrm{~m}^{3} / \mathrm{s}$ on évacuerait $10000 \mathrm{~kg} / \mathrm{s}$ (fig. 31 ).

Dans la partie aval, au contraire, les galets de vase ne pourront être transportés en quantité notable et on ne peut qu'espérer qu'ils seront dilués au cours des crues sur le premier tronçon de l'oued.

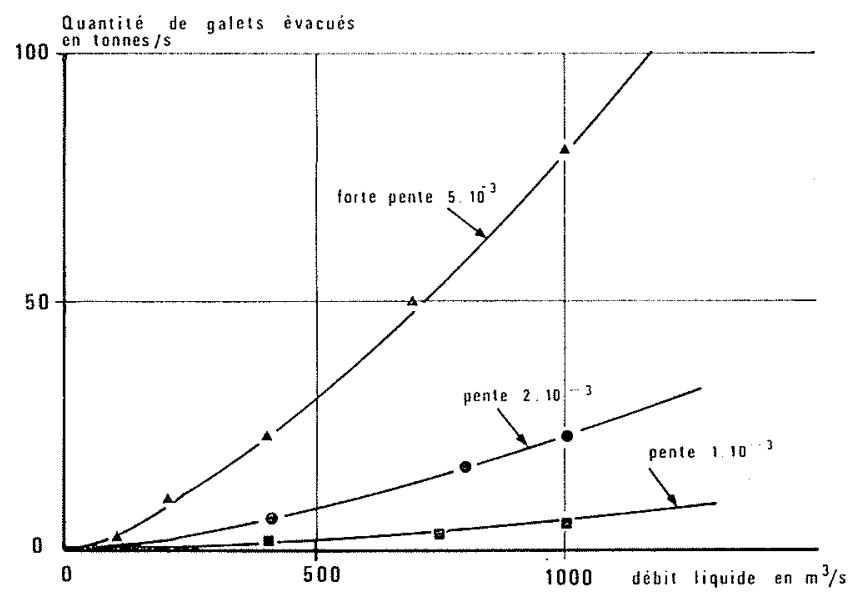

31/ Evacuation de galets de vase: valeurs calculées. 


\section{5 - Application pratique de cette étude. Possibilités de dragages dans la retenue du Hamiz}

Compte tenu des possibilités de transport de l'Oued Hamiz en aval du barrage, de la nature des matériaux, des apports d'eau dans la retenue et de leur utilisation par l'irrigation, il est possible de définir un programme de dragage et de chasse susceptible d'éviter un colmatage trop important de la rivière.

La drague susceptible d'être utilisée pour le dévasement du barrage a un débit de 1,25 à $1,6 \mathrm{~m}^{3} / \mathrm{s}$ et peut évacuer des mixtures à raison de 160 à $225 \mathrm{~kg} / \mathrm{s}$ pour les vases argileuses et 250 à $320 \mathrm{~kg} / \mathrm{s}$ pour les vases sableuses (les concentrations en sédiments refoulés varient de 13 à $20 \%$; ces concentrations correspondent à des volumes de vase en place divisés par des volumes de mixture refoulée).

Pour éviter que le barrage ne se trouve asséché, il faut en premier lieu limiter les dragages à la saison pluvieuse, qui s'étale de décembre à avril, en se basant sur un volume moyen de vase refoulée de 300000 à $350000 \mathrm{~m}^{3}$ par mois. Il serait possible, dans ces conditions, d'évacuer un débit liquide moyen de 5 à $6 \mathrm{~m}^{3} / \mathrm{s}$ par les vannes de fond.

Il est bien évident que ce chiffre devra être modulé en fonction de l'irrégularité des crues et qu'en fait les débits évacués varieront entre 1 et $10 \mathrm{~m}^{3} / \mathrm{s}$, entraînant des concentrations limites dans loued en aval du barrage comprises entre 15 et $300 \mathrm{~g} / 1$.

Pour ces caractéristiques de débits de mixtures, les études de la géomorphologie et les études théoriques de la capacité de transport de l'oued dans ses différents tronçons montrent que :

- En ce qui concerne les vases, il ne semble pas qu'il y ait de grands problèmes à condition d'adapter le débit liquide au débit de vase à transporter. Pour des débits supérieurs à $5 \mathrm{~m}^{3} / \mathrm{s}$ il doit être possible de transporter les mixtures de vases ayant une concentration inférieure à $300 \mathrm{~g} / 1$ jusqu'à la mer.

- Le problème est différent pour les sables et, si l'on peut transporter avec des débits de $10 \mathrm{~m}^{3} / \mathrm{s}$ des quantités de sable de $130 \mathrm{~kg} / \mathrm{s}(13 \mathrm{~g} / 1)$ dans la zone des fortes pentes, il faut s'attendre à un dépôt de ce type de matériau dans la zone des faibles pentes située en aval de la route nationale 5. Si l'on admet que les sables représentent $10 \%$ du volume des sédiments dragués, c'est-à-dire un volume de $150000 \mathrm{~m}^{3}$ par an, correspondant à un poids de 300000 tonnes de sable, il faudrait laisser déverser dans l'oued l'équivalent d'une crue de $100 \mathrm{~m}^{3} / \mathrm{s}$ pendant 11,5 jours, ce qui représenterait environ le double du volume disponible pendant toute une année, donc une impossibilité.

- Les galets et graviers se déposeront dès les premiers kilomètres en aval du barrage et il faudrait dépasser des débits de $100 \mathrm{~m}^{3} / \mathrm{s}$ pour les graviers et $300 \mathrm{~m}^{3} / \mathrm{s}$ pour les gros galets pour qu'ils puissent atteindre la partie aval de l'oued où ils se déposeront dans tous les cas. On ne peut donc compter, pour leur évacuation jusqu'à la mer, que sur leur usure, ce qui reste assez problématique.

- Les galets de vase ne pourront être entraînés que par des débits de crue dépassant $100 \mathrm{~m}^{3} / \mathrm{s}$ et uniquement dans la zone de forte pente. Pour être évacués jusqu'à la mer, il faudrait que ces galets de vase puissent se dégrader et se diluer au cours des dix premiers kilomètres de parcours. Il serait possible par exemple, en disposant de crue de $220 \mathrm{~m}^{3} / \mathrm{s}$ pendant quelques heures chaque mois, d'entraîner la majeure partie des galets de vase produits par la drague. Ici encore on constate que ce souhait est utopique et il faudra attendre vraisemblablement trois ou quatre ans après les dragages pour que l'oued se débarrasse de ses galets de vase en aval du barrage.

Cette étude de la capacité de transport de l'Oued Hamiz en aval du barrage, si elle ne permet pas de résoudre tous les problèmes qui peuvent se poser, apporte des éléments d'appréciation des risques de colmatage de l'oued par les différents matériaux et permet d'établir le planning des dragages en fonction des volumes d'eau disponibles. Compte tenu du pourcentage important de vase à évacuer, il apparaît qu'en répartissant les dragages sur trois à quatre années il doit être possible d'éviter des colmatages très importants de l'oued. Les travaux réalisés en nature, entre 1967 et 1970, semblent confirmer les résultats des études empiriques et théoriques effectuées par le LCHF et montrent qu'il est possible de prévoir l'influence du dragage d'une retenue de barrage sur l'environnement.

\section{Discussion}

Président : M. THÉvenIN

M. le Président remercie MM. Valembors et Migniot de leur originale communication et poursuit:

Avoir entendu parler du réservoir du Haniz m'a rajeuni d'une vingtaine d'années, puisque c'est au début des années 1950 qu'en A'gérie on a commencé à s'occuper sérieusement du dévasement des barrages; et c'est en 1955 que je suis a!lé à Lubeck recevoir la draguc suceuse qui a servi ail dévasement du Hamiz. Je pense que l'opération a été réussie grâce aux études auxquelles vous avez procédé.

Voici quelques chiffres qui montrent l'importance du problème du dévasement en Algérie:

En 1957, les barrages d'A'gérie, qui représentaient une capacité totale de 900 millions de metres cubes environ (grandes et moyennes retenues) avaient accumulé près de 200 millions de mètres cubes de vase; et, au rythme de l'envasement annuel, on pouvait prévoir que leur capacité serait réduite des deux tiers à la fin du siècle. Il fallait donc absolument prendre des mestires pour reconstituer leurs capacités utiles.

Si le problème de l'extraction et du transport des vases sur la longueur des retenues est relativement faci'e à résoudre par des procédés bien connus, l'évacuation des matériaux dans le lit (car on ne peut les stocker immédiatement à l'aval des barrages) pose de très gros problèmes en raison de la faiblesse de la puissance hydrau'ique de la plupart des oueds algériens; en dehors de crues quelquefois très espacées dans le temps, ceux-ci ne transitent, en effet, que de faibles débits.

On en était venu à l'idée (qui n'a pas donné lieu à des réalisations) de construire des barrages de chasse. On aurait doublé chaque retenue d'un réservoir de chasse destiné à créer des crues artificielles de gros débit pormettant d'évacuer, ou au moins de faire avancer, les matériaux déposés; ceux-ci comportent non seulement les vases - Souvent assez faci'ement entraînées par des débits relativement faibles - mais aussi les matériaux de granulométrie plus forte (sables et galets, galets de vase ou galets de pierre).

Je ne sais si ces problèmes ont été parfaitement maîtrisés dans le cas du Hamiz. Les disponibilités en eau, sans être importantes, devraient, ici, permettre d'évacuer au moins les matériaux les plus fins jusqu'à la mer. 